\title{
Pseudopartitive Nominalgruppen vom Typ Nquant+Adj+N in der deutschen Literatursprache des 17. bis 20. Jahrhunderts
}

\begin{abstract}
Der Aufsatz untersucht die grammatische Gestaltung zweigliedriger Nominalgruppen mit quantifizierendem nominalem Erstglied (Nquant) und quantifiziertem, durch ein Adjektivattribut erweitertem, nominalem Zweitglied (Adj+N), z.B. (mit) einem Glas kaltem Wasser. In der deutschen Gegenwartssprache ist in solchen Fügungen mit Varianten zu rechnen: (mit) einem Glas kalten Wassers, (mit) einem Glas kaltes Wasser. Insgesamt lassen sich fünf Konstruktionstypen unterscheiden. Anhand einer Belegsammlung aus literarischen Prosatexten vom 17. bis zum 20. Jahrhundert wird insbesondere die quantitative Verteilung von Konstruktionen mit Genitiv vs. Kasusübereinstimmung ins Auge gefasst. Anders als bei Nquant+N-Gruppen ohne Adjektivattribut im Zweitglied zeigt sich ein kräftiger Anstieg im Anteil der Genitivkonstruktion vom 17. bis zum 19. und nur ein leichter Rückgang im 20. Jahrhundert. Dieser Befund stimmt nur zum Teil mit den Darstellungen in der Standardliteratur überein. Eine mögliche Erklärung für die quantitative Entwicklung der Genitivkonstruktion in der Literatursprache liegt im Einfluss normativer Grammatiken.

This paper investigates the grammatical form of two-part noun phrases consisting of a quantifying first noun (Nquant) and a quantified second noun which is modified by an attributive adjective (Adj+N), e.g. (mit) einem Glas kaltem Wasser. In present-day German, noun phrases of this kind show some morphological variation: (mit) einem Glas kalten Wassers, (mit) einem Glas kaltes Wasser. On the whole, five types of constructions can be distinguished. The study focuses on the quantitative distribution of genitive vs. case agreement constructions in a corpus of seventeenth to twentieth century literary prose. In contrast to Nquant $+\mathrm{N}$ phrases without an adjective modifying the second noun, the proportion of the genitive construction is found to increase heavily from the seventeenth to the nineteenth century, diminishing only slightly in the twentieth. This finding corresponds only partly to the descriptions available in the standard literature. A possible explanation for the quantitative evolution of the genitive construction in literary language is the influence of normative grammar.
\end{abstract}

\section{Einleitung}

Der vorliegende Beitrag ${ }^{1}$ untersucht die Entwicklung zweigliedriger pseudopartitiver Nominalgruppen vom Typ Nquant+Adj+N (z.B. ein Glas kaltes Wasser) in literarischen Prosatexten vom 17. bis zum 20. Jahrhundert. Die untersuchten Nominalgruppen bestehen aus einem quantifizierenden nominalen Erstglied (Nquant) und einem quantifizierten, durch ein Adjektivattribut erweiterten, nominalen Zweitglied $(\operatorname{Adj}+\mathrm{N})$. Der Aufsatz ist wie folgt gegliedert.

In Kapitel 2 werden unterschiedliche Möglichkeiten der grammatischen Gestaltung von Nquant+Adj+N-Gruppen vorgestellt, wobei der Blick zunächst hauptsächlich auf die Gegenwartssprache gerichtet wird. Es wird eine Hypothese über die historische Entwicklung skizziert, die sich in der Grammatikliteratur andeutet und die im weiteren Verlauf des Aufsatzes

1 Die Hauptautorin ist Professorin für Deutsche Sprache an der Staatlichen Moskauer Universität für Internationale Beziehungen MGIMO. Der Beitrag stützt sich auf die für ihre Dissertation (Shubina 2000) und ihre Habilitationsschrift (Shubina 2007) erhobenen Daten. Der Koautor ist Wissenschaftler am Institut für Deutsche Sprache (IDS) Mannheim. Er hat bei der Auswertung und Interpretation des Belegkorpus, bei der Literaturschau sowie bei der Niederschrift in deutscher Sprache mitgewirkt. Die Autoren danken Bernd Wiese (IDS) sowie zwei anonymen Gutachtern für wertvolle Hinweise. 
überprüft wird. Kapitel 3 beschreibt die globale Entwicklung der Nquant+Adj+N-Gruppen vom 17. bis zum 20. Jahrhundert. In Kapitel 4 folgt eine detailliertere Untersuchung nach dem Kasus des Erstglieds, dem Genus bzw. Numerus des Zweitglieds und der Semantik des quantifizierenden Nomens. Kapitel 5 versucht eine abschließende Deutung.

Die Untersuchung stützt sich auf ein Korpus von 2.577 Belegen aus literarischen Texten repräsentativer Autoren aus der Zeit von 1645 bis 1990. Der Beginn dieses Zeitraums fällt noch in die frühneuhochdeutsche Periode, eine Phase ausgeprägter sprachlicher Variation, in der die neuhochdeutsche Schriftsprache sich allmählich herausbildete. Ab 1650 setzen die meisten Sprachhistoriker das eigentliche Neuhochdeutsch an (vgl. Keller 1986, S. 454ff.; Wells 1990, S. $283 \mathrm{ff}$.). Für das 18. und 19. Jhd. sind zunehmende Normierung und Systemstabilität bei abnehmender Variation kennzeichnend (vgl. Wells 1990, S. 366ff.). Vereinheitlichende Einflüsse gingen unter anderem von der Grammatikschreibung aus. In der zweiten Hälfte des 20. Jhds. ist eine allmähliche Schwächung der Normen zu beobachten, die möglicherweise künftig wieder zu verstärkter Variation führen wird. Hier wirkt sich zweifellos auch das vorwiegend deskriptive Selbstverständnis der gegenwärtigen Grammatikschreibung aus (zum Verhältnis zwischen Norm, System, Variation und Wandel in der (Gegenwarts-)Sprache vgl. Busse 2006; Konopka/Strecker 2009; Grabarek 2013).

Das untersuchte Textkorpus zum 17. Jhd. umfasst 19 Werke von Autoren wie Grimmelshausen, Gryphius, Santa Clara, Olearius, Schottelius und anderen. Es handelt sich um Autoren, die einen wichtigen Einfluss auf die in dieser Zeit stattfindende Normbildung der neuhochdeutschen Literatursprache hatten. Das Korpus zum 18. Jhd. besteht aus 59 Werken von Goethe, Schiller, Wieland, Musäus, Jacobi, Moritz, Campe und anderen. Das Korpus zum 19. Jhd. umfasst 80 Werke von Autoren wie Fontane, Freitag, Gutzkow, Heine, Immermann, Raabe, Storm und anderen. Aus der ersten Hälfte des 20. Jhds. wurden 125 Werke von Thomas und Heinrich Mann, Kafka, Hesse, Döblin, Kästner, Remarque, Seghers und anderen ausgewertet; aus der zweiten Hälfte des 20. Jhds. 218 Werke von Christa Wolf, Grass, Lenz, Walser, Böll, Handke, Konsalik, Strittmatter und anderen. Durchweg wurden unbearbeitete Ausgaben im Originalwortlaut verwendet. Die Belege wurden mangels geeigneter automatischer Extraktionsverfahren von Hand herausgesucht. Es wurde angestrebt, sämtliche enthaltenen Nquant+Adj+N-Gruppen zu erfassen.

\begin{tabular}{|c|c|}
\hline Zeitraum & Zahl der Belege \\
\hline 17. Jahrhundert & 144 Belege \\
\hline 18. Jahrhundert & 222 Belege \\
\hline 19. Jahrhundert & 448 Belege \\
\hline erste Hälfte des 20. Jahrhunderts & 819 Belege \\
\hline zweite Hälfte des 20. Jahrhunderts & 944 Belege \\
\hline Summe: & 2.577 Belege \\
\hline
\end{tabular}

Tab. 1: Überblick über das Belegkorpus

Die Ergebnisse der Untersuchung können nur für literarische Texte Gültigkeit beanspruchen. Daten aus anderen Funktionsbereichen sowie insbesondere aus der gesprochenen Alltagsrede wurden nicht einbezogen. Dazu müssen weitere empirische Studien folgen. 


\section{Problemstellung}

Nominalgruppen des Typs Nquant $+\mathrm{N}$ ohne Adjektivattribut im Zweitglied (z.B. ein Glas Milch) erlauben es im Gegenwartsdeutschen weder, über die Kasusform des Zweitglieds (hier: Milch), noch über die Art der grammatischen Verbindung zwischen Erst- und Zweitglied Schlussfolgerungen zu ziehen. Im Neuhochdeutschen wird bei femininen Substantiven wie Milch der Kasus formal nicht angezeigt. Bei Maskulina und Neutra wird er nur im Genitiv angezeigt (Wein-es, Wasser-s). Der Genitivanschluss, z.B. ein Glas Wassers, ist in Nquant+N-Gruppen ohne Adjektivattribut heute aber nicht mehr gebräuchlich. Im Mittelhochdeutschen war er ,sehr verbreitet“ (Paul 1919, S. 294), z.B. ein fuoder wines, zwo Unz Silbers, sechshundert Sekel Goldes. Bei Zweitgliedern im Maskulinum und Neutrum geht er ab dem 16. Jhd. kontinuierlich zurück: kain Unz Gold. Bei Zweitgliedern im Femininum kann er schon zu dieser Zeit nicht mehr markiert werden, weil die Genitivform der femininen Nomina bereits abgebaut ist. Nur im gewählten und poetischen Ausdruck bleibt der Genitiv in Nquant+N-Gruppen bis ins 19. Jhd. sporadisch erhalten (vgl. Blatz 1896, S. 374ff.; Erdmann/Mensing 1898, S. 101ff.; Behaghel 1923, S. 532f., S. 539f.; Wegera 1987; Hartweg/Wegera 1989, S. 117). In der Gegenwartssprache ist er so gut wie verschwunden.

Das bedeutet jedoch nicht, dass den Zweitgliedern in Nquant+N-Gruppen die Kasuskategorie fehlt. Die grammatischen Verhältnisse zeigen sich deutlicher, wenn im Zweitglied ein Adjektiv hinzugefügt wird: frisch-er Milch (Genitiv oder Dativ), rot-en Wein (Akkusativ), kalt-em Wasser (Dativ). In Nquant+Adj+N-Gruppen ist der Kasus des Zweitglieds auch in der Gegenwartssprache oft klar als Genitiv zu erkennen, z.B. ein Glas kalt-en Wasser-s, eine Menge geschliffen-er Steine. In anderen Fällen besteht Kasusübereinstimmung zwischen Erst- und Zweitglied, z.B. ein Glas kalt-es Wasser, eine Menge geschliffen-e Steine (Nominativ oder Akkusativ). Das Zweitglied kann aber auch in einer Form stehen, die weder als Genitiv markiert ist noch im Kasus mit dem Erstglied übereinstimmt, z.B. mit einem Eimer kaltes Wasser.

In Nquant+Adj+N-Gruppen kann das Zweitglied gegenwartssprachlich auf fünf Arten angefügt werden (so im Grundsatz schon dargestellt bei Adelung 1782, Bd. 2, S. 315f.):

(i) im Genitiv: mit einem Glas kalten Wassers

(ii) mit Kasusübereinstimmung und starker Adjektivdeklination: mit einem Glas kaltem Wasser

(iii) mit Kasusübereinstimmung und schwacher Adjektivdeklination: mit einem Glas kalten Wasser

(iv) weder im Genitiv noch mit Kasusübereinstimmung: mit einem Glas kaltes Wasser

(v) ohne Adjektivdeklination: mit einem Glas kalt Wasser

In Konstruktionen des Typs (i) ist das Zweitglied syntaktisch als Erweiterung zum Erstglied zu deuten. Ebenso ist das Verhältnis in präpositionalen Fügungen wie eine Reihe von alten Büchern, die in diesem Aufsatz nicht untersucht werden. In Konstruktionen der Typen (ii) bis (v) ist weniger klar, welches der beiden Glieder als Erweiterung zum anderen fungiert und welches Kern der Gesamtgruppe ist. Bei Kasusübereinstimmung (Typen ii und iii) kann das Zweitglied als Kern und das Erstglied als von ihm abhängiger Quantifikator gedeutet werden (so schon Becker 1870, Bd. 2, S. 135). Konstruktionen vom Typ 
(iii) kommen in der Gegenwartssprache nur sporadisch vor. Laut Duden (2001, S. 103) wird das Adjektiv im Zweitglied gewöhnlich stark dekliniert (ein Stück brüchiges Eisen). Nur nach Erstgliedern im Dativ tritt gelegentlich erkennbar die schwache Deklination auf. Das bestätigt sich in den untersuchten Texten aus dem 20. Jhd. (Beleg (1) mit neutralem Erstglied stammt nicht aus dem untersuchten Korpus):

(1) mit einem Stück selbst gebackenen Kuchen (Stern)

(2) Ich versuchte sie zu einer Flasche guten Wein einzuladen (Hesse)

In Konstruktionen der Typen (iv) und (v) ist die syntaktische Beziehung zwischen Erstund Zweitglied durch reine Angrenzung gekennzeichnet. Das Fehlen der Kasusübereinstimmung bei dekliniertem Adjektiv (Typ iv) lässt sich sicher feststellen, wenn das Erstglied im Dativ oder im Genitiv steht, z.B. von zwei Kisten geräucherte Flundern, mit einem Korb reife Äpfel. Die Form des Zweitglieds stimmt dann mit dem Nominativ überein. Eventuell kann sie im Sinne von Blatz (1896, S. 375), Erdmann/Mensing (1898, S. 102) und Erben (1965, S. 152) als neutrale, für Kasus unbestimmte Form gedeutet werden. Dieser Konstruktionstyp kommt vor allem bei Zweitgliedern im Plural vor. Mit Zweitglied im Singular, z.B. mit einem Sack guter Zement oder eines Sacks guter Zement, stufen Engel (1988, S. 618) und die Duden-Autoren (2001, S. 99) ihn als regelwidrig ein. In den untersuchten literarischen Texten kommen solche Belege nicht vor, aber in der Alltagsrede sind sie, wie auch Engel (1988, S. 618) feststellt, durchaus anzutreffen - mit femininem oder neutralem Zweitglied (mit einer Flasche fettarme Milch / kühles Bier) häufiger als mit maskulinem. Das Vorkommen von Konstruktionen des Typs (v) in der deutschen Sprachgeschichte ist z.B. von Trojanskaja (1972, S. 43 ff.) untersucht worden. Nach Paul (1919, S. 95ff.) waren unflektierte pränominale Adjektivattribute noch im Mittelhochdeutschen häufig, sind in neuhochdeutscher Zeit aber stark zurückgegangen. In Texten des 20. Jhds. findet sich Konstruktionstyp (v) noch vereinzelt als Archaismus mit stilistischer Sonderfunktion, aber nur wenn das Zweitglied ein Neutrum ist:

(3) Ich griff einen Kanten trocken Brot (Grass)

(4) dass nicht zufällig daraus ein Tropfen siedend Öl auf ihn falle (Th. Mann)

Geschichtlich scheint sich bei Nquant+Adj+N-Gruppen ein Wandel in der Bevorzugung von Konstruktionen des Typs (i) zu solchen des Typs (ii) vollzogen zu haben, analog zum Rückgang des Genitivanschlusses in Nquant+N-Gruppen ohne Adjektivattribut. Darauf deuten die Darstellungen in vielen Grammatiken hin (etwa Becker 1870, Bd. 2, S. 135; Blatz 1896, S. 374ff.; Paul 1919, S. 294ff.). Ende des 19. Jhds. wird Typ (i) von den meisten Autoren bevorzugt: Genitivanschluss gilt als die Regel, z.B. ein Scheffel guten Weizens (nicht: guter Weizen) (vgl. Andresen 1898, S. 262); Kasusübereinstimmung kommt in der Umgangssprache vor, z.B. eine Gesellschaft brave Leute (statt braver) (vgl. Erdmann/ Mensing 1898, S. 102). Abgeschwächt, aber ähnlich äußern sich zahlreiche Grammatiker noch in der Mitte des 20. Jhds.: Genitivanschluss ist in gepflegter Sprache die Regel, z.B. ein Glas guten Weines (nicht: guten Wein), bei einem Glase perlenden Sekts, ein Strau $\beta$ duftender Rosen (vgl. Jung 1954, S. 350; Erben 1965, S. 91; Hirschbold 1962, S. 69; Brinkmann 1971, S. 13); Kasusübereinstimmung kommt in der Geschäfts- und Umgangssprache vor, ist aber nicht gefordert (vgl. Engel 1929, S. 255; Betcke 1947, S. 53). Ab der zweiten Hälfte des 20. Jhds. wird Typ (ii) bevorzugt. Genitivanschluss wird „,zunehmend durch die enge Apposition oder durch präpositionale Attribute verdrängt: eine Tasse duftenden Kaffees (dafür oft duftender Kaffee, mit duftendem Kaffee)“, außer bei plurali- 
schem Gemessenem (eine Gruppe junger Leute) (Flückiger 1988, S. 161). „Wo ein feierlicher Rahmen gegeben ist, da ist der Genitiv auch heute noch [...] stilistisch besser als der Nominativ.“ (Hallwass 1979, S. 350). Kasusübereinstimmung ist die Regel; bei bestimmten Konstruktionen ist der Genitiv aber akzeptabel: ein Liter kühlen Wassers (vgl. Löbel 1986, S. 52ff.). Die Untersuchung in den Kapiteln 3 und 4 des vorliegenden Aufsatzes wird ein differenzierteres Licht auf die historische Entwicklung werfen und - zumindest für die Literatursprache - zu einer modifizierten Sicht führen.

Betrachten wir zuvor noch genauer die Situation in der Gegenwartssprache. Laut Duden (2001, S. 590; 2009, S. 984ff.) wird gegenwartssprachlich nach Erstgliedern im Nominativ und Akkusativ gewöhnlich Kasusübereinstimmung mit starker Adjektivdeklination (Typ ii) gewählt, ebenso nach Erstgliedern im Dativ vor Zweitgliedern im Singular: ein Zentner kanadischer Weizen, nach 3 Glas heißem Rotwein. Nur noch vereinzelt und in gewählter Rede werde das Zweitglied in den Genitiv gesetzt (Typ i): bei einer Tasse duftenden Kaffees. Wenn das Erstglied im Dativ und das Zweitglied im Plural steht, werde aber noch häufig der Genitivanschluss gewählt: der Erlös aus einem Zentner lagerfähiger Äpel. Alternativ werde auf den Nominativ ausgewichen bzw. auf die Kasusmarkierung des Zweitglieds verzichtet (Typ iv): der Erlös aus einem Zentner lagerfähige Äpfel. Typ (ii) sei dann unüblich: ?der Erlös aus einem Zentner lagerfähigen Äpfeln. Empirisch sind Beispiele mit Kasusübereinstimmung jedoch auch für diesen Fall leicht zu finden: aus 60 Hektar eigenen Weinbergen, einem Dutzend ausländischen Investoren in Quatar (Frankfurter Allgemeine Zeitung).

Außer vom Kasus des Erstglieds und vom Numerus des Zweitglieds hängt die Wahl des Konstruktionstyps von der Bedeutung des quantifizierenden Nomens ab. Nach einem Einheitennomen (einem Substantiv, das eine Maßeinheit bezeichnet, wie Eimer, Flasche, Fass, Fuder, Handvoll, Packen, Tropfen usw.) richtet sich ein singularisches Zweitglied im Kasus nach dem Erstglied (Typ ii bzw. iii). Seltener kommt Genitivanschluss vor (Typ i) (vgl. Duden 2009, S. 984ff.):

Nominativ: $\quad$ eine Tasse schwarzer Kaffee wird dir gut tun (Typ ii) 'eine Tasse schwarzen Kaffees wird dir gut tun (Typ i)

Akkusativ: ich schenke dir eine Tasse schwarzen Kaffee ein (Typ ii/iii) ?ich schenke dir eine Tasse schwarzen Kaffees ein (Typ i)

Dativ: $\quad$ mit einer Tasse schwarzem Kaffee (Typ ii) mit einer Tasse schwarzen Kaffees (Typ i)

Genitiv: $\quad$ der Genuss einer Tasse schwarzen Kaffees (Typ ii/i)

Steht nach einem Einheitennomen das Zweitglied im Plural, so sind die Konstruktionstypen (i) bis (iv) möglich:

Nominativ: dort steht ein Korb frische Äpfel (Typ ii/iv) dort steht ein Korb frischer Äpfel (Typ i)

Akkusativ: Otto brachte einen Korb frische Äpfel (Typ ii/iv)

Otto brachte einen Korb frischer Äpfel (Typ i)

Dativ: $\quad$ mit einem Korb frischen Äpfeln (Typ ii/iii)

mit einem Korb frischer Äpfel (Typ i)

mit einem Korb frische Äpfel (Typ iv) 
Genitiv: $\quad$ der Saft eines Korbes frischer Äpfel (Typ ii/i) der Saft eines Korbes frische Äpfel (Typ iv)

Nach Sammelbezeichnungen (Substantiven, die eine Mehrzahl von Individuen bezeichnen, wie Anzahl, Gruppe, Reihe, Schwarm, Truppe usw.) steht das Zweitglied durchweg im Plural und häufig im Genitiv (Typ i). Daneben kommen Typ (ii) bzw. (iii) und (iv) vor:

Nominativ: ein Rudel hungriger Wölfe streifte durch den Wald (Typ i) ein Rudel hungrige Wölfe streifte durch den Wald (Typ ii/iv)

Akkusativ: $\quad$ es gibt eine Anzahl großer Unternehmen (Typ i) es gibt eine Anzahl große Unternehmen (Typ ii/iv)

Dativ: $\quad$ man setzt sich einer Myriade stechlustiger Insekten aus (Typ i) man setzt sich einer Myriade stechlustige Insekten aus (Typ iv) man setzt sich einer Myriade stechlustigen Insekten aus (Typ ii/iii)

Genitiv: $\quad$ mit Hilfe eines Trupps externer Berater (Typ i/ii)

Einige Autoren vertreten die Meinung, dass die Semantik noch weiter gehende Auswirkungen auf die grammatische Gestaltung von Nquant+Adj+N-Gruppen hat. So sind etwa Löbel (1986, S. 53ff.) zufolge in der Gegenwartssprache bei Sammelbezeichnungen, wenn das Zweitglied für nicht-belebte Gegenstände steht, sowohl Kasusübereinstimmung als auch Genitivanschluss akzeptabel: ein Stapel bunte Illustrierte, ein Stapel bunter Illustrierter. Werden dagegen Gruppen von Personen quantifiziert, so sei Genitivanschluss gegenüber Kasusübereinstimmung zu bevorzugen: eine Bande vernachlässigter Jugendlicher gegenüber eine Bande vernachlässigte Jugendliche. Diese Beobachtung bestätigt sich in unseren Daten. Bei Sammelbezeichnungen, die selbst nicht zählbar sind (*drei Anzahlen), sind Kasusübereinstimmung oder Anschluss ohne Kasusangleichung nach Löbel (ebd.) bevorzugt gegenüber dem Genitiv: eine Menge ausländische Reisende gegenüber eine Menge ausländischer Reisender. Diese These wird von unseren Daten nicht bestätigt.

Im untersuchten Korpus zeigt sich aber, dass bei den Sammelbezeichnungen eine andere semantische Unterscheidung relevant ist. Nach Sammelbezeichnungen im engeren Sinne wie Gruppe, Horde, Rudel, Schar, Schwarm, Trupp usw. steht das Zweitglied fast ausschließlich im Genitiv (Typ i). Kasusübereinstimmung (Typen ii und iii) ist bei Erstgliedern dieser Subklasse selten. Für das Gegenwartsdeutsche findet sie sich im Korpus nur in Texten von Günter Grass (z.B. ein Grüppchen schlesische Neusiedler, mehrere Reihen uniformierte Parteigenossen, einen Stoß aufzuhaltende Langhölzer, ein biersaures Bündel geschlitzte und blutverkrustete Uniformen). Dagegen sind bei Zahlnomina (quantifizierenden Nomina mit unmittelbar numerischer Bedeutung wie Paar, Dutzend, Hundert usw.) gleichermaßen Konstruktionen der Typen (i) und (ii) möglich.

Wir unterscheiden daher im weiteren Verlauf zwischen Einheitennomina (Nquant1), Sammelbezeichnungen im engeren Sinne (Nquant2) und Zahlnomina (Nquant3). Auf Nquant1 kann ein Zweitglied im Singular (Nquant1a) oder Plural (Nquant1b) folgen. Auf Nquant2 und Nquant3 kann das quantifizierte Nomen nur im Plural folgen. 


\section{Globale Entwicklung der Nquant+Adj+N-Gruppen vom 17. bis zum 20. Jahrhundert}

Wir geben nun anhand des Belegkorpus zunächst einen Überblick über die Verteilung von Nquant+Adj+N-Gruppen mit Nquantla bis Nquant3 auf die Konstruktionstypen (i) bis (v) vom 17. bis zum 20. Jhd.

Bei der quantitativen Auswertung sind einige formale Mehrdeutigkeiten zu berücksichtigen. Steht beispielsweise das Erstglied im Genitiv, so kann die Nominalgruppe mit gleich guten Gründen als Genitivanschluss (Typ i) oder als Kasusübereinstimmung mit starker Adjektivdeklination (Typ ii) gedeutet werden (vgl. Blatz 1896, S. 376):

(5) wegen eines Pakets grauer Nudeln (Fallada)

(6) mit Hülfe eines großen Stücks brauner Leinewand (Wieland)

(7) in Erwartung eines größeren Stücks freien Wassers (Lenz)

Steht das Erstglied im Dativ und trägt das Adjektiv bei femininem Zweitglied die Endung $-r$, so ist ebenfalls nicht entscheidbar, ob Genitivanschluss (Typ i) oder Kasusübereinstimmung (Typ ii) vorliegt:

(8) zu einigen großen Schalen saurer Milch (Gutzkow)

(9) wischte mit einem Klumpen alter Putzwolle (Frank)

Steht das Erstglied im Akkusativ und ist das Zweitglied ein Maskulinum, so besteht formal kein Unterschied zwischen starker und schwacher Adjektivdeklination (Typ ii bzw. iii):

(10) Die haben mir drei Tassen heißen Kaffee eingebracht ... (Storm)

Formübereinstimmung zwischen starker und schwacher Adjektivdeklination (Typ ii und iii) besteht auch beim Erstglied im Dativ und Zweitglied im Plural:

(11) zu etlichen Dutzet kleinen Pastetlin (Grimmelshausen)

(12) mit einem Paar kleinen Augen (Wieland)

(13) Wir haben das Wort von hundert Millionen amerikanischen Bürgern (Kellermann)

Steht vor einem Zweitglied im Plural das Erstglied im Nominativ oder Akkusativ, so kann die Nominalgruppe Typ (ii) (Kasusübereinstimmung mit starker Adjektivflexion) oder Typ (iv) (weder Genitiv noch Kasusübereinstimmung) zugeordnet werden:

(14) worauf drei Reihen eiserne Töpfe standen (Heine)

Steht das Erstglied im Nominativ oder Akkusativ und ist das Zweitglied ein Femininum, so erlaubt die Nominalgruppe sogar drei Deutungen - als Typ (ii), (iii) oder (iv):

(15) ein klein Schälchen warme Suppe (Heine)

(16) darin sie einen Ballen frische Butter trug (Grimmelshausen)

Alle Belege, die zwei formale Deutungen erlauben, werden bei der quantitativen Auswertung den möglichen Konstruktionstypen je zur Hälfte zugeordnet; Belege, die drei Deutungen erlauben, werden je zu einem Drittel gezählt. Tabellen 2 bis 6 zeigen die Verteilung der Belege auf die Konstruktionstypen: 


\begin{tabular}{|l|l|l|l|l|l|}
\hline & Nquant1a & Nquant1b & Nquant2 & Nquant3 & Summe \\
\hline Typ (i) & $13,5(18 \%)$ & $11(46 \%)$ & $8(80 \%)$ & $11(32 \%)$ & $43,5(30 \%)$ \\
\hline Typ (ii) & $16,3(20,5 \%)$ & $6,5(26 \%)$ & $1(10 \%)$ & $10,5(31 \%)$ & $34,3(24 \%)$ \\
\hline Typ (iii) & $18,8(25 \%)$ & $1(4 \%)$ & - & $1(3 \%)$ & $20,8(15 \%)$ \\
\hline Typ (iv) & $0,3(0,5 \%)$ & $5,5(24 \%)$ & $1(10 \%)$ & $9,5(28 \%)$ & $16,3(11 \%)$ \\
\hline Typ (v) & $27(36 \%)$ & - & - & $2(6 \%)$ & $29(20 \%)$ \\
\hline Summe & $76(100 \%)$ & $24(100 \%)$ & $10(100 \%)$ & $34(100 \%)$ & $144(100 \%)$ \\
\hline Anteil & $53 \%$ & $17 \%$ & $7 \%$ & $23 \%$ & $100 \%$ \\
\hline
\end{tabular}

Tab. 2: Verteilung der Konstruktionstypen im Korpus zum 17. Jhd.

\begin{tabular}{|l|l|l|l|l|l|}
\hline & Nquant1a & Nquant1b & Nquant2 & Nquant3 & Summe \\
\hline Typ (i) & $26,5(33 \%)$ & $37(90 \%)$ & $21(91 \%)$ & $19(25 \%)$ & $103,5(46 \%)$ \\
\hline Typ (ii) & $29(38 \%)$ & $2(5 \%)$ & $1(4,5 \%)$ & $30,5(38 \%)$ & $62,5(28 \%)$ \\
\hline Typ (iii) & $6,5(8 \%)$ & $0,5(1 \%)$ & - & $3,5(4,5 \%)$ & $10,5(5 \%)$ \\
\hline Typ (iv) & $2(2 \%)$ & $1,5(4 \%)$ & $1(4,5 \%)$ & $26(32,5 \%)$ & $30,5(14 \%)$ \\
\hline Typ (v) & $15(19 \%)$ & - & - & - & $15(7 \%)$ \\
\hline Summe & $79(100 \%)$ & $41(100 \%)$ & $23(100 \%)$ & $79(100 \%)$ & $222(100 \%)$ \\
\hline Anteil & $36 \%$ & $18 \%$ & $10 \%$ & $36 \%$ & $100 \%$ \\
\hline
\end{tabular}

Tab. 3: Verteilung der Konstruktionstypen im Korpus zum 18. Jhd.

\begin{tabular}{|l|l|l|l|l|l|}
\hline & Nquant1a & Nquant1b & Nquant2 & Nquant3 & Summe \\
\hline Typ (i) & $90,5(57 \%)$ & $81(90 \%)$ & $104(99 \%)$ & $32(34 \%)$ & $307,5(68 \%)$ \\
\hline Typ (ii) & $50,3(32 \%)$ & $4,5(5 \%)$ & $0,5(0,5 \%)$ & $32(34 \%)$ & $87,3(20 \%)$ \\
\hline Typ (iii) & $12,8(8 \%)$ & $0,5(0,5 \%)$ & - & $5,5(6 \%)$ & $18,8(4 \%)$ \\
\hline Typ (iv) & $1,3(1 \%)$ & $4(4,5 \%)$ & $0,5(0,5 \%)$ & $25,5(26 \%)$ & $31,3(7 \%)$ \\
\hline Typ (v) & $3(2 \%)$ & - & - & - & $3(1 \%)$ \\
\hline Summe & $158(100 \%)$ & $90(100 \%)$ & $105(100 \%)$ & $95(100 \%)$ & $448(100 \%)$ \\
\hline Anteil & $35 \%$ & $20 \%$ & $24 \%$ & $21 \%$ & $100 \%$ \\
\hline
\end{tabular}

Tab. 4: Verteilung der Konstruktionstypen im Korpus zum 19. Jhd.

\begin{tabular}{|l|l|l|l|l|l|}
\hline & Nquant1a & Nquant1b & Nquant2 & Nquant3 & Summe \\
\hline Typ (i) & $175(47 \%)$ & $136(91 \%)$ & $77(100 \%)$ & $120(54,5 \%)$ & $508(62 \%)$ \\
\hline Typ (ii) & $148,2(40 \%)$ & $7,5(5 \%)$ & - & $50,5(23 \%)$ & $206,2(25 \%)$ \\
\hline Typ (iii) & $42,2(11 \%)$ & - & - & $1(0,5 \%)$ & $43,2(5,5 \%)$ \\
\hline Typ (iv) & $5,7(1,5 \%)$ & $6,5(4 \%)$ & - & $48,5(22 \%)$ & $60,7(7,5 \%)$ \\
\hline Typ (v) & $1(0,5 \%)$ & - & - & - & $1(0 \%)$ \\
\hline Summe & $372(100 \%)$ & $150(100 \%)$ & $77(100 \%)$ & $220(100 \%)$ & $819(100 \%)$ \\
\hline Anteil & $45 \%$ & $18 \%$ & $10 \%$ & $27 \%$ & $100 \%$ \\
\hline
\end{tabular}

Tab. 5: Verteilung der Konstruktionstypen im Korpus zur ersten Hälfte des 20. Jhds.

\begin{tabular}{|l|l|l|l|l|l|}
\hline & Nquant1a & Nquant1b & Nquant2 & Nquant3 & Summe \\
\hline Typ (i) & $186(37 \%)$ & $192(90 \%)$ & $86,5(96 \%)$ & $66(45 \%)$ & $530,5(56,5 \%)$ \\
\hline Typ (ii) & $213,2(44 \%)$ & $10,5(5 \%)$ & $2(2 \%)$ & $40(27,5 \%)$ & $265,7(28 \%)$ \\
\hline Typ (iii) & $71,2(14 \%)$ & $0,5(0 \%)$ & - & - & $71,7(7,5 \%)$ \\
\hline Typ (iv) & $19,7(4 \%)$ & $10(5 \%)$ & $1,5(2 \%)$ & $40(27,5 \%)$ & $71,2(7,5 \%)$ \\
\hline Typ (v) & $5(1 \%)$ & - & - & - & $5(0,5 \%)$ \\
\hline Summe & $495(100 \%)$ & $213(100 \%)$ & $90(100 \%)$ & $146(100 \%)$ & $944(100 \%)$ \\
\hline Anteil & $52 \%$ & $23 \%$ & $10 \%$ & $15 \%$ & $100 \%$ \\
\hline
\end{tabular}

Tab. 6: Verteilung der Konstruktionstypen im Korpus zur zweiten Hälfte des 20. Jhds. 
In allen Teilkorpora sind die vier Subklassen von Erstgliedern (Nquant1a bis 3) vertreten. Bis auf das Teilkorpus aus dem 19. Jhd. stellen Sammelbezeichnungen (Nquant2) überall die kleinste Beleggruppe. In allen Teilkorpora zeigt sich bei ihren Zweitgliedern die geringste Formvariation. Die Gruppen mit Zweitglied im Singular (Nquant1a) stellen zwischen einem Drittel und gut der Hälfte der Belege. Sie zeigen fast durchweg mehr Variation als die Gruppen mit Zweitglied im Plural (Nquant1b, 2 und 3). Nur im 17. Jhd. zeigen Gruppen mit Nquant3 genauso viel Variation. Konstruktionstyp (v) (ohne Adjektivdeklination) ist bis auf zwei Vorkommen mit Nquant3 aus dem 17. Jhd. nur mit Nquant1a belegt, jedoch nur im 17. und 18. Jhd. in nennenswerter Anzahl. Danach ist Typ (v) marginal. Typ (iv) ist in Nquant1a-Gruppen durchweg sehr schwach vertreten. In Gruppen mit pluralischem Zweitglied (Nquant1a, 2 und 3) ist sein Anteil höher.

In der Summe haben in allen fünf Teilkorpora die Konstruktionen mit Genitivanschluss (Typ i) die größten Anteile, gefolgt von Konstruktionen mit Kasusübereinstimmung und starker Adjektivdeklination (Typ ii). Die Anteile der Typen (iii) und (iv) sind in allen Teilkorpora deutlich kleiner.

Bei Typ (i) zeigt sich vom 17. bis zum 19. Jhd. eine Zunahme von weniger als einem Drittel der Belege auf mehr als zwei Drittel. Der Genitivanschluss ist demnach offenbar nicht einfach der ältere Konstruktionstyp, der in der Gegenwart ersetzt wird. Er wurde in der Literatursprache vielmehr erst in neuhochdeutscher Zeit (wieder) dominant, wahrscheinlich zum Teil unter dem Einfluss sprachnormerischer Bemühungen. So schreibt etwa Schottelius (1663, Bd. 1, S. 712): ,Wenn zusammen kommen zwey selbstendig Nennwörter [d.h. Substantive] / alsdann muß das eine in der Geschlechtsendung [d.h. im Genitiv] gesetzet werden.“ Bei Gottsched (1762, S. 417) heißt es: „Wenn zwey oder mehr Hauptwörter zusammen kommen, die weder ein und, noch ein oder verbindet; so stehen eins oder mehrere allemal in der zweyten Endung [d.h. im Genitiv].“ Adelung (1782, Bd. 2, S. 315f.) schreibt speziell über den von uns untersuchten Konstruktionstyp: „Im Oberdeutschen gebraucht man [...] häufig den Genitiv, welchen oft die höhere Schreibart auch im Hochdeutschen nachahmet, eine Summe Geldes, mit einem Stücke Brotes [...] Nothwendig wird oft der Genitiv, wenn der Classen-Nahme noch ein Bestimmungswort vor sich hat, ein Gericht seltener Fische, oder der seltensten Fische [...]". Deskriptiv werden diese Verallgemeinerungen durch unsere Daten aus dem 17. und 18. Jhd. nicht bestätigt. Es dürfte sich vielmehr um normativ gemeinte Aussagen handeln, die in den zeitgenössischen Diskurs um die sich herausbildende neuhochdeutsche Standard-Schriftsprache einzuordnen sind (zu diesem Diskurs vgl. die detaillierte Darstellung von Hundt 2000).

Im 20. Jhd. zeigt sich ein Rückgang des Genitivanschlusses, der aber viel geringer ausfällt als in Nquant+N-Gruppen ohne Adjektiv und auch geringer, als man aufgrund der Darstellungen in der Grammatikliteratur erwarten würde. Typ (i) ist mit deutlich über 50\% der Belege in der zweiten Hälfte des 20. Jhds. noch doppelt so stark vertreten wie Typ (ii). Zumindest in literarischen Texten scheint er für Nquant+Adj+N-Gruppen demnach weiterhin der dominante Konstruktionstyp zu sein.

Die Anteile von Konstruktionstyp (ii) liegen dagegen in den Teilkorpora nahezu konstant bei ca. einem Viertel der Belege. Nur im 19. Jhd. sind sie etwas niedriger. Die Zunahme im 20. Jhd. führt zum gleichen Prozentsatz zurück, den Typ (ii) bereits im 18. Jhd. hatte. Wenn wir die Typen (ii) und (iii) zusammenfassen, wird deutlicher, dass Nquant+Adj+NGruppen mit Kasusübereinstimmung bereits im 17. Jhd. einen recht hohen Anteil hatten, 
und zwar höher als in der 2. Hälfte des 20. Jhds. Im 17. Jhd. waren sie sogar häufiger als Typ (i). Erst ab dem 18. Jhd. hat Typ (i) konstant einen wesentlich höheren Anteil als die Typen (ii) und (iii) zusammen. Bis zum 19. Jhd. geht Kasusübereinstimmung zurück; im 20. Jhd. nimmt ihr Anteil wieder zu, erreicht aber nicht annähernd den Anteil des Genitivanschlusses.

Genitivanschluss und Kasusübereinstimmung zusammen haben im Korpus zum 17. Jhd. einen Anteil von gut zwei Dritteln. Daneben ist vor allem Typ (v) (ohne Adjektivdeklination) mit einem Fünftel der Belege noch stark vertreten. Auch Typ (iv) (weder Genitiv noch Kasusübereinstimmung) hat mit $11 \%$ noch einen nennenswerten Anteil. Im 18. und 19. Jhd. findet - zumindest in den untersuchten literarischen Texten - eine nicht übersehbare Vereinheitlichung statt. Genitivanschluss und Kasusübereinstimmung werden zu den dominanten Konstruktionsalternativen, deren Anteil auf zusammen über 90\% ansteigt und in der Summe dann bis zur 2. Hälfte des 20. Jhds. konstant bleibt. Konstruktionen mit reiner Angrenzung von Erst- und Zweitglied (weder Genitiv noch Kasusübereinstimmung) sinken zu einer eher unbedeutenden dritten Variante ab. Erhalten bleibt in dieser Gruppe nicht der im 17. Jhd. stärkere Konstruktionstyp (v), sondern Typ (iv), dessen Anteil über den gesamten Untersuchungszeitraum hinweg relativ stabil ist. Tabelle 7 fasst die Beobachtungen bis hierher zusammen:

\begin{tabular}{|l|c|c|c|c|c|}
\hline & 17. Jhd. & 18. Jhd. & 19. Jhd. & 20. Jhd. 1 & 20. Jhd. 2 \\
\hline Typ (i) & $30 \%$ & $46 \%$ & $68 \%$ & $62 \%$ & $56,5 \%$ \\
\hline Typ (ii) & $24 \%$ & $28 \%$ & $20 \%$ & $25 \%$ & $28 \%$ \\
\hline Typen (ii)+(iii) & $39 \%$ & $33 \%$ & $24 \%$ & $30,5 \%$ & $35,5 \%$ \\
\hline Typen (i)+(ii)+(iii) & $69 \%$ & $79 \%$ & $92 \%$ & $92,5 \%$ & $92 \%$ \\
\hline Typen (iv)+(v) & $31 \%$ & $21 \%$ & $8 \%$ & $7,5 \%$ & $8 \%$ \\
\hline
\end{tabular}

Tab. 7: Verteilung von Genitivanschluss (i), Kasusübereinstimmung (ii/iii) und reiner Angrenzung (iv/v) in den fünf Untersuchungszeiträumen

\section{Morphologische Gestaltung der Nquant+Adj+N-Gruppen vom 17. bis zum 20. Jahrhundert}

Im Folgenden schauen wir Nquant+Adj+N-Gruppen mit den vier Subklassen quantifizierender Nomina im Detail an. Dabei wird sich zeigen, dass die historische Entwicklung bei den Nquant-Klassen teilweise sehr unterschiedlich verlaufen ist.

4.1 Konstruktionen mit Einheitennomina und Zweitglied im Singular (Nquant1a)

Das Korpus enthält 1.180 Belege mit Nquantla. Das entspricht einem Anteil von 46\%. Es wurde schon darauf hingewiesen, dass diese Gruppen eine höhere konstruktionelle Varianz zeigen als Gruppen mit pluralischem Zweitglied. Das scheint damit zusammenzupassen, dass die Singularformen auch morphologisch diversifizierter sind als die Pluralformen.

Über alle fünf Untersuchungszeiträume hinweg sind bei Nquantla die Konstruktionstypen (i) bis (v) im Korpus belegt, was bei pluralischem Zweitglied nirgends der Fall ist. Die Hälfte bis zwei Drittel der Erstglieder stehen im Akkusativ. Die Zweitglieder sind Kontinuativa (nicht-zählbare Massenomina). Sie stehen für unbelebte Entitäten, die in Akkusativsprachen syntaktisch bevorzugt im Objekt erscheinen. Etwa vier Fünftel der Zweitglieder sind Maskulina oder Neutra. Nur in der zweiten Hälfte des 20. Jhds. liegt der Anteil der femininen Zweitglieder über 20\%. 


\subsubsection{Jahrhundert}

Gut die Hälfte der Belege aus dem 17. Jhd. (76: 53\%) hat ein Zweitglied im Singular: 31 von ihnen (41\%) haben ein maskulines, 37 (49\%) ein neutrales, $8(10 \%)$ ein feminines Zweitglied. Das Erstglied steht in 69\% der Belege im Akkusativ, in 18\% im Dativ, in 13\% im Nominativ. Belege mit genitivischem Erstglied fehlen. Tabelle 8 schlüsselt die Beleggruppe nach Konstruktionstyp, Kasus des Erstglieds und Genus des Zweitglieds auf:

\begin{tabular}{|l|c|c|c|c|c|c|c|c|c|c|}
\hline Erstglied & \multicolumn{3}{|c|}{ Nominativ } & \multicolumn{3}{c|}{ Akkusativ } & \multicolumn{3}{c|}{ Dativ } & Summe \\
\hline Zweitglied & $\mathrm{m}$ & $\mathrm{n}$ & $\mathrm{f}$ & $\mathrm{m}$ & $\mathrm{n}$ & $\mathrm{f}$ & $\mathrm{m}$ & $\mathrm{n}$ & $\mathrm{f}$ & \\
\hline Typ (i) & - & - & 1 & - & 7 & 2 & - & 3 & 0,5 & $13,5(18 \%)$ \\
\hline Typ (ii) & 2 & 1 & 0,3 & 10,5 & 1 & 1 & - & - & 0,5 & $16,3(20,5 \%)$ \\
\hline Typ (iii) & - & - & 0,3 & 10,5 & - & 1 & 6 & 1 & - & $18,8(25 \%)$ \\
\hline Typ (iv) & - & - & 0,3 & - & - & & - & - & - & $0,3(0,5 \%)$ \\
\hline Typ (v) & 1 & 3 & 1 & 1 & 18 & - & - & 3 & - & $27(36 \%)$ \\
\hline Summe & 3 & 4 & 3 & 22 & 26 & 4 & 6 & 7 & 1 & $76(100 \%)$ \\
\hline Anteil & \multicolumn{3}{|c|}{$10(13 \%)$} & \multicolumn{3}{|c|}{$52(69 \%)$} & \multicolumn{3}{|c|}{$14(18 \%)$} & $76(100 \%)$ \\
\hline
\end{tabular}

Tab. 8: Verteilung der Belege mit Nquant1a aus dem 17. Jhd.

Belege vom Typ (i) (Zweitglied im Genitiv) bilden knapp ein Fünftel dieser Gruppe. Die Erstglieder stehen im Akkusativ oder im Dativ:

(17) daß einer für eine Summe geliehen Geldes einen Garten, Acker oder sonst was versetzet und dafür gebrauchen lässet ... (Olearius)

(18) Dann neben seinen grossen Summen baares Geldes hat es hin und wieder viel Häuser, Land und Leute (Olearius)

Nur ein Beleg hat ein Erstglied im Nominativ:

(19) Gewiß ist es, daß es auch Sinnreiche geschnitzte Bilder an sich gehabt, welche gleich wie sie ein Theil großer Kunst, also waren sie auch die Ursach grosser Verwunderung (Stieler)

Im Zweitglied kommen ausschließlich Neutra und Feminina vor. Genitivisch angeschlossene Maskulina sind im Korpus erst ab dem 18. Jhd. belegt.

Bei den Neutra trägt in der Mehrzahl der Belege sowohl das Adjektiv als auch das Nomen das Genitiv-s (vgl. Ratke 1616, S. 58):

(20) bei Vermehrung einer Straffe von zwölf Marck lötiges Goldes (Schupp)

Nur selten trägt das Adjektiv die schwache Endung - $n$ :

(21) mit einer Bissel weißen Meels (Santa Clara)

Auch Konstruktionen mit unflektiertem Adjektiv, in denen nur das Substantiv die GenitivEndung trägt, kommen vor:

(22) ich weiß als nicht, was vor einen Hauffen gut Dings (Grimmelshausen)

Belege vom Konstruktionstyp (ii) machen ein Fünftel dieser Gruppe aus. Ihre Erstglieder stehen im Akkusativ oder im Nominativ:

(23) als die blosse Form, welche mehr als tausend Stücklein ... färbiges, zusammen gesetztes oder durch vielfältiges flicken aneinander genähtes Tuch noch vor Augen stellte (Grimmelshausen) 
(24) Ach in der Schnur Gasse, im wilden Mann ist ein köstlicher guter Trunk rother Wein (Schupp)

Der einzige Beleg mit Erstglied im Dativ kann auch Typ (i) zugerechnet werden:

(25) In dem wir aber über dieselbe mit 5. Stunden saurer Arbeit uns gewunden ... (Olearius)

Im Zweitglied sind alle drei Genera vertreten, aber Maskulina überwiegen bei weitem:

(26) packte ein stück faulen Käse (Grimmelshausen)

Belege vom Typ (iii) stellen ein Viertel der Gruppe. Die Erstglieder stehen im Akkusativ oder Dativ:

(27) Unsere Gesandten liessen ihm ihren Gruß entbieten, eine Flasche Französischen Brandwein verehren ... (Olearius)

(28) daß sie Ihr eine Nehnadel mit einem Faden weissen Zwirn bringen muste (Reuter)

Der einzige Beleg mit Erstglied im Nominativ kann auch Typ (ii) oder (iv) zugeordnet werden:

(29) als eine grosse Menge unglaubliche Schönheit an seiner Geliebten ihn aufzurichten (Stieler)

Als Zweitglieder finden sich ganz überwiegend Maskulina. Bei Belegen mit femininem Zweitglied ist alternativ die Zuordnung zu Typ (ii) möglich:

(30) darin sie einen Ballen frische Butter trug (Grimmelshausen)

Nur ein Beleg hat ein Neutrum als Zweitglied:

(31) fanden wir den Tisch schon bedeckt und mit einem Botthast, einem stück kalten Rindfleisch aus dem Salz geziert (Grimmelshausen)

Beleg (29) ist der einzige aus diesem Teilkorpus, der Konstruktionstyp (iv) zugeordnet werden kann. Bei singularischem Zweitglied ist Typ (iv) in allen untersuchten Zeiträumen sehr selten. Bei pluralischem Zweitglied kommt er weitaus häufiger vor. Alle Belege vom Typ (iv) mit Nquantla haben ein feminines Zweitglied nach einem Erstglied im Nominativ oder Akkusativ. Sie können alternativ auch den Typen (ii) oder (iii) zugeordnet werden. Man kann das so deuten, dass Typ (iv) bei singularischem Zweitglied real nicht vorkommt.

Konstruktionstyp (v) (mit nicht-dekliniertem Adjektiv) ist mit mehr als einem Drittel der Belege der häufigste in diesem Teilkorpus. Die Erstglieder stehen im Akkusativ oder Nominativ, seltener im Dativ:

(32) und er bekam zur Verehrung 9. Ellen braun Tuch (Olearius)

(33) das waren vier und siebenzig Marck ungemüntzt fein Silber (Grimmelshausen)

(34) mit allerhand Stückern weiß Brod (Grimmelshausen)

Die Zweitglieder sind ganz überwiegend Neutra. Maskuline und vor allem feminine Zweitglieder sind selten:

(35) werde ein guter Trunk bitter Wein sehr gesund sein (Schupp)

(36) grosse stücken geronnen Milch sich finden (Olearius) 
Die flexivlose Form dominiert bei einsilbigen Adjektiven: lange Ellen fein roth Tuch, ein Stück gut Geld, ein Faß frisch Wasser, ein Stück schwartz Brot. Ferner tritt sie bei Stoffadjektiven mit dem $n$-Suffix auf: ein Stück silbern Tobin, etliche Stück seiden zeug, ein Balle Wüllen Tuch, bei Partizipien: 12 grosse Stück geräuchert Fisch, ein Stück stinkent Wildpret sowie bei Ableitungen mit dem Suffix -isch: ein hauffen närrisch Zeug, zwey Stück Persianisch Seidenzeug.

Für die Kasus der Erstglieder und die Genera und Numeri der Zweitglieder zeigen die Belege deutlich unterschiedliche Konstruktionspräferenzen.

Erstglieder im Akkusativ kommen anteilig etwa gleich häufig mit den Konstruktionstypen (i), (ii), (iii) und (v) vor. Erstglieder im Nominativ bevorzugen die Konstruktionstypen (ii) (Kasusübereinstimmung mit starker Adjektivdeklination) und (v) (ohne Adjektivdeklination). Erstglieder im Dativ bevorzugen die Typen (iii) (Kasusübereinstimmung mit schwacher Adjektivdeklination) und (i) (Genitivanschluss). Daneben kommt - anteilig seltener - Typ (v) vor. Da Erstglieder im Nominativ und Dativ nur mit relativ wenigen Belegen im Korpus vertreten sind, muss die sich andeutende teilweise komplementäre Verteilung mit Vorsicht betrachtet werden.

Deutlicher sind die Unterschiede bei den Genera der Zweitglieder. Maskuline Zweitglieder bevorzugen die Konstruktionstypen mit Kasusübereinstimmung (ii/iii). Genitivanschluss (Typ i) fehlt bei ihnen im 17. Jhd. noch ganz; Typ (v) (ohne Adjektivdeklination) ist selten. Neutrale Zweitglieder bevorzugen dagegen Typ (v) - fast zwei Drittel der Belege - oder (i) - knapp ein Drittel. Bei ihnen ist Kasusübereinstimmung die Ausnahme. Bei den wenigen Belegen mit femininem Zweitglied zeigen sich keine Konstruktionspräferenzen, zumal die Konstruktionstypen hier teilweise nicht unterscheidbar sind.

Die Gesamtverteilung bei singularischem Zweitglied (Nquantla) ist deutlich anders als bei pluralischem Zweitglied (Nquant1b, 2 und 3; siehe Unterkapitel 4.2 bis 4.4). Die Konstruktionstypen (i) und (ii) (überwiegend bzw. durchweg mit starker Adjektivdeklination), die in den späteren Untersuchungszeiträumen einen immer größeren Anteil haben, machen im 17. Jhd. nur gut die Hälfte der Belege aus, bei Nquantla sogar deutlich weniger. Dagegen kommen die Typen (iii) (mit schwacher Adjektivdeklination) und (v) (ohne Adjektivdeklination), die in den späteren Untersuchungszeiträumen immer seltener werden, im 17. Jhd. noch sehr häufig vor, und zwar gerade in Wortgruppen mit singularischem Zweitglied. Hier machen sie mehr als 60\% der Belege aus. Einen so hohen Anteil haben sie in keinem anderen Teilkorpus.

Man kann diese Beobachtung mit der generell großen Variationsbreite in Zusammenhang bringen, die das Frühneuhochdeutsche als sprachgeschichtliche Übergangsphase kennzeichnet (vgl. Keller 1986, S. 326ff., 398ff.; Wells 1990, S. 244ff.; Hartweg/Wegera 1989). Im speziellen Bezug auf die grammatische Struktur von Nquant+Adj+N-Gruppen deutet sie auf eine variable, oft unbestimmte Verteilung der Kern- und Attributrollen hin. Genitivischer Anschluss, der das Erstglied als Kern und das Zweitglied als Erweiterung erscheinen lässt, hat dabei den geringsten Anteil. Kasusübereinstimmung, die dem Zweitglied mehr Gewicht gibt und zulässt, es als Kern der Konstruktion zu deuten, hat den größten Anteil, vor allem bei maskulinem Zweitglied, macht aber insgesamt weniger als die Hälfte der Belege aus. Einen großen Anteil, insbesondere bei neutralem Zweitglied, haben Fügungen, die die Kern- und Attributrollen unbestimmt lassen. 


\subsubsection{Jahrhundert}

Die absolute Zahl der Belege mit Nquantla aus dem 18. Jhd. ist mit 79 etwa genauso groß wie die aus dem 17. Jhd. Ihr Anteil am Teilkorpus für den Untersuchungszeitraum ist mit $36 \%$ aber geringer, d.h. für das 18. Jhd. sind relativ mehr Belege mit pluralischem Zweitglied vorhanden. 18 Belege (23\%) haben ein maskulines, 46 (58\%) ein neutrales, 15 (19\%) ein feminines Zweitglied. Das Erstglied steht in 66\% im Akkusativ, in 18\% im Nominativ, in $15 \%$ im Dativ, in $1 \%$ im Genitiv:

\begin{tabular}{|l|c|c|c|c|c|c|c|c|c|c|c|c|c|}
\hline Erstglied & \multicolumn{3}{|c|}{ Nominativ } & \multicolumn{3}{|c|}{ Akkusativ } & \multicolumn{3}{c|}{ Dativ } & \multicolumn{3}{c|}{ Genitiv } & Summe \\
\hline Zweitglied & m & n & f & m & n & f & m & n & f & m & n & f & \\
\hline Typ (i) & 1 & 1 & 2 & 6 & 3 & 4 & 4 & 4 & 1 & - & - & 0,5 & $26,5(33 \%)$ \\
\hline Typ (ii) & 2 & 4 & 0,7 & 2,5 & 17 & 1,3 & - & - & 1 & - & - & 0,5 & $29(38 \%)$ \\
\hline Typ (iii) & - & - & 0,7 & 2,5 & - & 1,3 & - & 2 & - & - & - & - & $6,5(8 \%)$ \\
\hline Typ (iv) & - & - & 0,7 & - & - & 1,3 & - & - & - & - & - & - & $2(2 \%)$ \\
\hline Typ (v) & - & 2 & - & - & 13 & - & - & - & - & - & - & - & $15(19 \%)$ \\
\hline Summe & 3 & 7 & 4 & 11 & 33 & 8 & 4 & 6 & 2 & - & - & 1 & $79(100 \%)$ \\
\hline Anteil & \multicolumn{1}{|c|}{$14(18 \%)$} & $52(66 \%)$ & \multicolumn{1}{|c|}{$12(15 \%)$} & \multicolumn{1}{|c|}{$1(1 \%)$} & $79(100 \%)$ \\
\hline
\end{tabular}

Tab. 9: Verteilung der Belege mit Nquant1a aus dem 18. Jhd.

Belege vom Typ (i) machen in diesem Teilkorpus 33\% aus. Das entspricht annähernd einer Verdopplung ihres Anteils gegenüber dem 17. Jhd.:

(37) wozu ein begleitender Trunk ausgesuchten Weines zum allerbesten schmeckte (Goethe)

Der Genitivanschluss kommt jetzt nicht mehr nur bei femininen und neutralen, sondern auch bei maskulinen Zweitgliedern vor, sogar mit überdurchschnittlichem Anteil. Während insgesamt 23\% der Belege maskuline Zweitglieder haben, sind es bei Typ (i) über $40 \%$ (11 von 26,5). Der Anteil der Neutra als Zweitglieder ist dagegen mit 30\% gegenüber 58\% stark unterdurchschnittlich. Die Erstglieder stehen im Akkusativ oder Dativ, seltener im Nominativ:

(38) einen Trog frischen Kalks (Goethe)

(39) der lange nach einem Tropfen frischen Wassers schmachtete (Wieland)

Der einzige Beleg mit Erstglied im Genitiv kann als Typ (i) oder (ii) analysiert werden:

(40) mit Hilfe eines Stücks brauner Leinewand (Wieland)

Auch die Belege vom Typ (ii) haben sich gegenüber dem 17. Jhd. auf 38\% fast verdoppelt. Die Erstglieder stehen im Akkusativ oder Nominativ:

(41) Er packte also ein Pfund guten Tee ... (Stilling)

(42) Du kommst mir vor, wie ein Pack gut gekämmte Spanische Wolle oder fein gesponnenes Garn ... (Nikolai)

Nur ein Beleg hat ein Erstglied im Dativ:

(43) nach einigen Tagen gütlicher Pflege (Goethe)

Bei den Zweitgliedern sind alle drei Genera vertreten.

Typ (iii) ist gegenüber dem 17. Jhd. stark zurückgegangen. Die meisten Belege dieser Gruppe können alternativ als Typ (ii) analysiert werden. Bei den wenigen eindeutigen Fällen steht das Erstglied im Dativ; das Zweitglied ist ein Neutrum: 
(44) mit 400 Pfund Sterl. baren Gelde (Schnabel)

Konstruktionstyp (iv) ist nur mit wenigen Belegen vertreten, die alternativ als Typ (ii) oder (iii) analysiert werden können. Ihr Erstglied steht im Nominativ oder Akkusativ; das Zweitglied ist ein Femininum:

(45) daß er selbiges Tages ein Stück heilige Arbeit gefunden hatte (Schnabel)

Konstruktionstyp (v) geht vom 17. zum 18. Jhd. um etwa die Hälfte zurück. Er kommt jetzt nur noch in Gruppen mit Erstglied im Akkusativ oder selten im Nominativ und einem Neutrum im Zweitglied vor:

(46) als wenn mir ein Kübel eiskalt Wasser übern Nacken spritze (Schiller)

Die Mehrzahl dieser Belege stammt von Johann Gottfried Schnabel, der sich darin offenbar am älteren Sprachgebrauch orientiert (10. Thlr. klein Geld, etliche Ballen weiß Papier, ein Stück verschimmelt Brot, einen Trunk frisch Wasser u.a.).

Gegenüber dem 17. Jhd. macht das Korpus teilweise gleiche, teilweise aber auch veränderte Konstruktionspräferenzen deutlich.

Erstglieder im Akkusativ kommen weiterhin relativ ausgeglichen mit den Konstruktionstypen (i), (ii), (iii) und (v) vor. Typ (v) ist bei ihnen überdurchschnittlich, Typ (i) leicht unterdurchschnittlich vertreten. Die Verteilung ist hier noch der im 17. Jhd. ähnlich. Erstglieder im Nominativ bevorzugen die Konstruktionstypen (i), (ii) und (v). Typ (ii) und vor allem Typ (i) sind gegenüber dem 17. Jhd. deutlich erstarkt; Typ (v) ist zurückgegangen. Bei Erstgliedern im Dativ zeigt sich das Anwachsen von Konstruktionstyp (i) am klarsten. Hier weisen $75 \%$ der Belege einen Genitivanschluss auf. Demgegenüber ist Konstruktionstyp (iii) stark zurückgegangen; Typ (v) ist verschwunden. Auch in diesem Teilkorpus sind aber nur relativ wenige Belege mit Erstglied im Nominativ und Dativ enthalten, sodass die Verteilungen mit Zurückhaltung interpretiert werden müssen.

Maskuline Zweitglieder bevorzugen jetzt den Konstruktionstyp (i) (Genitiv: mehr als 60\% der Belege) vor (ii) (Kasusübereinstimmung mit starker Adjektivdeklination: 25\%). Fünf Belege mit Erstglied im Akkusativ können alternativ als Typ (ii) oder (iii) gedeutet werden. Typ (v) (nicht-dekliniertes Adjektiv) ist bei maskulinem Zweitglied nicht mehr vertreten. Neutrale Zweitglieder bevorzugen die Typen (ii) (46\%) und (v) (33\%). Der Anteil von Typ (v) hat sich allerdings halbiert. Belege mit Genitivanschluss (17\%) sind ebenfalls nur noch halb so stark vertreten wie im 17. Jhd. Bei den Wortgruppen mit maskulinem und neutralem Zweitglied zeigt sich wiederum eine entgegengesetzte Verteilung, jedoch mit einer Verschiebung bei den Konstruktionstypen. Maskuline Zweitglieder treten jetzt häufiger als Genitiv-Attribute auf, neutrale als mögliche Wortgruppenkerne.

Belege mit femininem Zweitglied sind in diesem Teilkorpus knapp doppelt so häufig vertreten wie im Korpus aus dem 17. Jhd. Bei ihnen erkennt man ähnlich wie bei den Maskulina eine Präferenz für den Genitivanschluss (50\% der Belege) und die Kasusübereinstimmung mit starker Adjektivdeklination (23\%). Bei einigen Belegen ist alternativ eine Zuordnung zu den Typen (iii) oder (iv) möglich.

Auch im 18. Jhd. weicht die Verteilung bei singularischem Zweitglied (Nquant1a) von der bei pluralischem Zweitglied (Nquant1b, 2 und 3) ab. Genitivanschluss hat zwar zugenommen, ist aber bei Nquant1a noch im gleichen Maße wie im 17. Jhd. unterdurchschnittlich vertreten. Typ (v) ist zurückgegangen, hat aber nach wie vor einen weit überdurchschnittli- 
chen Anteil. Bei pluralischem Zweitglied kommt er ab dem 18. Jhd. gar nicht mehr vor. Die Konstruktionstypen (ii/iii) (mit Kasusübereinstimmung) sind wie im 17. Jhd. zusammen leicht überrepräsentiert. Allerdings ist der Anteil von Typ (iii) (mit schwacher Adjektivdeklination) zurückgegangen und der von Typ (ii) (mit starker Adjektivdeklination) angewachsen.

Im Gesamtbild zeigt sich eine stärkere Profilierung der Kern- und Attributrollen durch die Auswahl des Konstruktionstyps. Die Nquant+Adj+N-Gruppen zeigen mehr interne hierarchische Struktur. Die Konstruktionstypen, die die interne Struktur unbestimmt lassen, gehen zurück.

\subsubsection{Jahrhundert}

Die absolute Zahl der Korpusbelege mit Nquant1a aus dem 19. Jhd. ist mit 158 etwa doppelt so groß wie die aus dem 17. und 18. Jhd. Ihr Anteil am Teilkorpus für den Untersuchungszeitraum ist mit 35\% etwa genauso groß wie für das 18. Jhd. 69 Belege (44\%) haben ein maskulines, 61 (38\%) ein neutrales, 28 (18\%) ein feminines Zweitglied. Das Erstglied steht in 54\% der Belege im Akkusativ, in 26\% im Nominativ und in $20 \%$ im Dativ. Belege mit genitivischem Erstglied fehlen:

\begin{tabular}{|l|c|c|c|c|c|c|c|c|c|c|}
\hline Erstglied & \multicolumn{3}{|c|}{ Nominativ } & \multicolumn{3}{c|}{ Akkusativ } & \multicolumn{3}{c|}{ Dativ } & Summe \\
\hline Zweitglied & $\mathrm{m}$ & $\mathrm{n}$ & $\mathrm{f}$ & $\mathrm{m}$ & $\mathrm{n}$ & $\mathrm{f}$ & $\mathrm{m}$ & $\mathrm{n}$ & $\mathrm{f}$ & \\
\hline Typ (i) & 8 & 10 & 4 & 19 & 11 & 13 & 12 & 10 & 3,5 & $90,5(57 \%)$ \\
\hline Typ (ii) & 11 & 7 & 0,3 & 9,5 & 18 & 1 & - & - & 3,5 & $50,3(32 \%)$ \\
\hline Typ (iii) & - & - & 0,3 & 9,5 & - & 1 & - & 2 & - & $12,8(8 \%)$ \\
\hline Typ (iv) & - & - & 0,3 & - & - & 1 & - & - & - & $1,3(1 \%)$ \\
\hline Typ (v) & - & 1 & - & - & 2 & - & - & - & - & $3(2 \%)$ \\
\hline Summe & 19 & 17 & 5 & 38 & 31 & 16 & 12 & 12 & 7 & $158(100 \%)$ \\
\hline Anteil & \multicolumn{3}{|c}{$42(26 \%)$} & \multicolumn{3}{|c|}{$85(54 \%)$} & \multicolumn{3}{c|}{$31(20 \%)$} & $158(100 \%)$ \\
\hline
\end{tabular}

Tab. 10: Verteilung der Belege mit Nquant1a aus dem 19. Jhd.

In diesem Teilkorpus stellen Konstruktionen vom Typ (i) weit über die Hälfte der Belege. Gegenüber dem 18. Jhd. hat sich ihr Anteil erneut fast verdoppelt. Die Erstglieder stehen im Akkusativ, Nominativ oder Dativ; bei den Zweitgliedern sind alle drei Genera vertreten:

(47) darin sich ein Stückchen mittelalterlicher Wegelagerei erhalten hat (Fontane)

(48) trank bei Tische eine Flasche edlen Weins (Hoffmann)

(49) nach einem Glase frischen Wassers (Raabe)

Bei maskulinen und neutralen Zweitgliedern wird das Adjektiv jetzt ausschließlich mit der schwachen Genitivendung $-n$ realisiert.

Konstruktionstyp (ii), der im 17. und 18. Jhd. stärker als Typ (i) vertreten war, ist leicht zurückgegangen. Er macht jetzt nur noch ein knappes Drittel der Belege aus. Die Erstglieder stehen im Akkusativ oder Nominativ:

(50) um ein Glas frische Milch zu holen (Fontane)

(51) Da ist auch ein Bündel getrocknetes Fleisch ... (Raabe)

Die Belege mit Erstglied im Dativ sind alternativ als Typ (i) analysierbar:

(52) mit einer Tasse süßer Milch (Hoffmann) 
Die Zweitglieder sind überwiegend Neutra oder Maskulina:

(53) daß ein Stückchen rohes Fleisch und ... in Verbindung stehen können (Gutzkow)

(54) ein Glas guten Wein zu genießen (Hoffmann)

Konstruktionstyp (iii) ist gegenüber dem 18. Jhd. nicht zurückgegangen, und auch seine Verteilung hat sich kaum verändert. Wiederum sind fast alle Belege dieses Typs alternativ als Typ (ii) deutbar. Nur zwei Belege mit Erstglied im Dativ und einem Neutrum im Zweitglied sind eindeutig Typ (iii) zuzuordnen:

(55) mit einem Stück brüchigen Eisen (Raabe)

Typ (iv) bleibt marginal. Die wenigen Belege können alternativ als Typ (ii) oder (iii) analysiert werden.Typ (v) kommt nur noch in drei Belegen vor. Die Erstglieder stehen im Akkusativ oder Nominativ, die Zweitglieder sind Neutra:

(56) noch ein Tropfen niedersächsisch Blut durch die Adern rinnt (Raabe)

Die Daten zeigen die Fortsetzung einer recht eindeutigen Entwicklung vom 17. bis zum 19. Jhd. Der Genitivanschluss wird von einer eher seltenen Variante mit Schwerpunkt bei Akkusativ und Dativ im Erstglied und Neutrum im Zweitglied zum dominanten Konstruktionstyp für alle Kasus und Genera. Typ (v) mit nicht-dekliniertem Adjektiv, der im 17. Jhd. den größten Anteil hatte, allerdings mit Spezialisierung auf Neutra im Zweitglied, wird marginal. Kasusübereinstimmung hat einen relativ konstanten Anteil, aber starke Adjektivdeklination (Typ ii) nimmt zu, schwache (Typ iii) geht zurück. Typ (iv) ist im ganzen Zeitraum marginal.

Erstglieder im Akkusativ zeigen weiterhin eine größere Variation bei den Konstruktionstypen als die übrigen Kasus. Bei Typ (i) sind sie mit 47\% gegenüber 54\% leicht unterrepräsentiert. Erstglieder im Nominativ kommen praktisch nur noch mit den Konstruktionstypen (i) und (ii) vor. Erstglieder im Dativ bevorzugen wie schon im 18. Jhd. stark den Genitivanschluss (jetzt 82\% der Belege).

Maskuline Zweitglieder bevorzugen weiterhin den Konstruktionstyp (i) (57\% der Belege) vor (ii) (30\%). 19 Belege mit Erstglied im Akkusativ können alternativ als Typ (ii) oder (iii) gedeutet werden. Auch neutrale Zweitglieder bevorzugen jetzt Konstruktionstyp (i) (51\% der Belege) vor (ii) (41\%). Typ (v) ist stark zurückgegangen. Die unterschiedliche Verteilung der Konstruktionstypen bei maskulinem und neutralem Zweitglied, die sich im 17. und 18. Jhd. gezeigt hatte, besteht damit nicht mehr. Auch bei femininem Zweitglied ist Genitivanschluss wie schon im 18. Jhd. der bevorzugte Konstruktionstyp, mit 73\% der Belege sogar besonders deutlich. Typ (ii) ist bei ihnen mit $17 \%$ schwächer vertreten. Im 19. Jhd. haben sich damit Genitivanschluss (Typ i) und Kasusübereinstimmung mit starker Adjektivdeklination (Typ ii) bei den Nquantla+Adj+N-Gruppen als die hauptsächlichen Konstruktionsalternativen stabilisiert. Bei nominativischem und akkusativischem Erstglied stehen sie gleich stark nebeneinander. Bei dativischem Erstglied ist der Genitivanschluss eindeutig dominant.

Wiederum weicht die Verteilung bei singularischem Zweitglied (Nquant1a) von der bei pluralischem Zweitglied (Nquant1b, 2 und 3) ab. Bei singularischem Zweitglied ist der Genitivanschluss weniger dominant; Kasusübereinstimmung ist relativ stärker vertreten. 


\subsubsection{Erste Hälfte des 20. Jahrhunderts}

Das Teilkorpus aus der ersten Hälfte des 20. Jhds. ist mit 372 Nquant1a-Belegen mehr als doppelt so groß wie das zum 19. Jhd. Der Anteil am Gesamtkorpus für den Untersuchungszeitraum ist mit $45 \%$ wieder etwas größer. 141 Belege (38\%) haben ein maskulines, 159 (43\%) ein neutrales, 72 (19\%) ein feminines Zweitglied. Das Erstglied steht in $53 \%$ der Belege im Akkusativ, in 30\% im Nominativ und in 17\% im Dativ. Belege mit genitivischem Erstglied fehlen:

\begin{tabular}{|l|c|c|c|c|c|c|c|c|c|c|}
\hline Erstglied & \multicolumn{3}{|c|}{ Nominativ } & \multicolumn{3}{c|}{ Akkusativ } & \multicolumn{3}{c|}{ Dativ } & Summe \\
\hline Zweitglied & $\mathrm{m}$ & $\mathrm{n}$ & $\mathrm{f}$ & $\mathrm{m}$ & $\mathrm{n}$ & $\mathrm{f}$ & $\mathrm{m}$ & $\mathrm{n}$ & $\mathrm{f}$ & \\
\hline Typ (i) & 16 & 25 & 22 & 22 & 26 & 25 & 17 & 18 & 4 & $175(47 \%)$ \\
\hline Typ (ii) & 17 & 30 & 0,7 & 30,5 & 48 & 5 & 4 & 9 & 4 & $148,2(40 \%)$ \\
\hline Typ (iii) & - & - & 0,7 & 30,5 & - & 5 & 4 & 2 & - & $42,2(11 \%)$ \\
\hline Typ (iv) & - & - & 0,7 & - & - & 5 & - & - & - & $5,7(1,5 \%)$ \\
\hline Typ (v) & - & 1 & - & - & - & - & - & - & - & $1(0,5 \%)$ \\
\hline Summe & 33 & 56 & 24 & 83 & 74 & 40 & 25 & 29 & 8 & $372(100 \%)$ \\
\hline Anteil & \multicolumn{3}{|c|}{$113(30 \%)$} & \multicolumn{3}{|c|}{$197(53 \%)$} & \multicolumn{3}{c|}{$62(17 \%)$} & $372(100 \%)$ \\
\hline
\end{tabular}

Tab. 11: Verteilung der Belege mit Nquantla aus der ersten Hälfte des 20. Jhds.

Gegenüber dem Korpus aus dem 19. Jhd. ist der Anteil der Belege vom Typ (i) um 10 Prozentpunkte zurückgegangen. Ihre Erstglieder stehen im Akkusativ, Nominativ oder Dativ; bei den Zweitgliedern sind alle drei Genera vertreten:

$$
\text { ... eine Büchse guter dänischer Butter trägt (Traven) }
$$

(58) auf der Heizung stand eine halbgeleerte Tasse kaltgewordenen Tees (Feuchtwanger)

(59) mit einem breiten Laib selbstgebackenen Brotes (Koeppen)

Die Belege vom Typ (ii) haben gegenüber dem 19. Jhd. um 8 Prozentpunkte zugenommen. Hier kommen jetzt neben Erstgliedern im Akkusativ und Nominativ auch solche im Dativ vor, allerdings noch mit geringerem Anteil. Bei den Zweitgliedern sind auch hier alle drei Genera vertreten:

(60) ich bestellte mir eine dreifache Portion süße Speise (Fallada)

(61) Es war im Hofe eine große Ladung buchenes Holz angefahren (Hesse)

(62) Der Hauptgewinn besteht aus ... eindreiviertel Pfund magerem Speck (Kästner)

Die Belege vom Typ (iii) können in der Mehrzahl alternativ dem Typ (ii) zugerechnet werden. Nur sechs Belege mit Erstglied im Dativ und einem Maskulinum oder Neutrum im Zweitglied gehören eindeutig zu Typ (iii):

(63) Hier saß er ... bei einer Flasche leichten Mosel (Kellermann)

Die wenigen Belege vom Typ (iv) können ausnahmslos auch den Typen (ii) oder (iii) zugerechnet werden. Typ (v) ist nur noch mit einem einzigen Beleg vertreten.

Nquantla+Adj+N-Gruppen mit Erstglied im Akkusativ zeigen in der ersten Hälfte des 20. Jhds. keine größere konstruktionelle Varianz mehr als solche mit Erstglied in einem anderen Kasus. Beim Genitivanschluss sind sie mit einem Anteil von 42\% gegenüber 53\% weiterhin unterrepräsentiert; bei den Konstruktionen mit Kasusübereinstimmung ist ihr Anteil mit 63\% überdurchschnittlich. Gruppen mit Erstglied im Nominativ zeigen eine 
leichte, solche mit Erstglied im Dativ weiterhin eine deutliche Bevorzugung für den Genitivanschluss.

Maskuline und neutrale Zweitglieder bevorzugen jetzt Kasusübereinstimmung (61\% bei den Maskulina, 55\% bei den Neutra) gegenüber dem Genitivanschluss (39\% bzw. 43\%). Bei den femininen Zweitgliedern überwiegt wie schon im 18. und 19. Jhd. der Genitivanschluss: $71 \%$ gegenüber 29\%. In den Gruppen mit singularischem Zweitglied ist der Genitivanschluss in der ersten Hälfte des 20. Jhds. wieder deutlich seltener als in Gruppen mit pluralischem Zweitglied; Kasusübereinstimmung ist relativ stärker vertreten.

Die Daten aus der ersten Hälfte des 20. Jhds. zeigen damit teilweise eine Fortsetzung von früher beobachteten Entwicklungstendenzen, teilweise eine Umorientierung. Die Konzentration auf Genitivanschluss versus Kasusübereinstimmung als Konstruktionsalternativen für Nquant1 a+Adj+N-Gruppen verstärkt sich weiter. Wenn man aber die Belege der Typen (ii) und (iii) zusammenfasst, so zeigt sich, dass Kasusübereinstimmung mit einem Anteil von 51\% nun wieder stärker vertreten ist als der Genitivanschluss. Diese Entwicklung verstärkt sich in der zweiten Hälfte des 20. Jhds.

\subsubsection{Zweite Hälfte des 20. Jahrhunderts}

Das Teilkorpus aus der zweiten Hälfte des 20. Jhds. ist mit 495 Nquantla-Belegen das umfangreichste. Der Anteil am Gesamtkorpus für den Untersuchungszeitraum beträgt 52\%. 195 Belege (39\%) haben ein maskulines, 173 (35\%) ein neutrales, 127 (26\%) ein feminines Zweitglied. Das Erstglied steht in 65\% im Akkusativ, in 18\% im Nominativ, in $16 \%$ im Dativ und in $1 \%$ im Genitiv:

\begin{tabular}{|l|c|c|c|c|c|c|c|c|c|c|c|c|c|}
\hline Erstglied & \multicolumn{2}{|c|}{ Nominativ } & \multicolumn{3}{|c|}{ Akkusativ } & \multicolumn{3}{c|}{ Dativ } & \multicolumn{3}{c|}{ Genitiv } & Summe \\
\hline Zweitglied & m & n & f & m & n & f & m & n & f & m & n & f & \\
\hline Typ (i) & 4 & 15 & 5 & 43 & 27 & 42 & 19 & 19 & 10,5 & - & 1,5 & - & $186(37 \%)$ \\
\hline Typ (ii) & 24 & 27 & 4,3 & 45,5 & 71 & 15,3 & 9 & 5 & 10,5 & - & 1,5 & - & $213,2(44 \%)$ \\
\hline Typ (iii) & - & - & 4,3 & 45,5 & - & 15,3 & 5 & 1 & - & - & - & - & $71,2(14 \%)$ \\
\hline Typ (iv) & - & - & 4,3 & - & - & 15,3 & - & - & - & - & - & - & $19,7(4 \%)$ \\
\hline Typ (v) & - & - & - & - & 4 & - & - & 1 & - & - & - & - & $5(1 \%)$ \\
\hline Summe & 28 & 42 & 18 & 134 & 102 & 88 & 33 & 26 & 21 & - & 3 & - & $495(100 \%)$ \\
\hline Anteil & \multicolumn{3}{|c|}{$(18 \%)$} & \multicolumn{3}{|c|}{$324(65 \%)$} & \multicolumn{3}{|c|}{$80(16 \%)$} & \multicolumn{3}{|c|}{$3(1 \%)$} & $495(100 \%)$ \\
\hline
\end{tabular}

Tab. 12: Verteilung der Belege mit Nquantla aus der zweiten Hälfte des 20. Jhds.

Der Anteil der Belege vom Typ (i) hat sich gegenüber der ersten Hälfte des 20. Jhds. erneut um 10 Prozentpunkte reduziert. Allerdings fällt der Rückgang des Genitivanschlusses nicht so deutlich aus, wie man es nach den in Kapitel 2 zitierten Grammatikerurteilen erwarten könnte. Immer noch beträgt sein Anteil mehr als ein Drittel der Belege. Bei den Erstgliedern sind alle Kasus, bei den Zweitgliedern alle Genera vertreten:

(64) ... riss er bei jedem Vorüberflug eine Portion duftgetränkter Luft in sich hinein (Süskind)

(65) Vor dem Tor brannte ein Berg beschriebenen Papiers (Heiduczek)

(66) bei einem Glas gereiften Weins (Böll)

(67) in Erwartung eines größeren Stücks freien Wassers (Lenz) 
Bei den Belegen vom Typ (ii) zeigt sich gegenüber der ersten Hälfte des 20. Jhds. eine weitere Zunahme um 4 Prozentpunkte. Auch hier sind bei den Erstgliedern alle Kasus, bei den Zweitgliedern alle Genera vertreten:

(68) Ich bestellte beim Kellner ein Stück gedeckte Apfeltorte (Maron)

(69) Eine Flasche bulgarischer Rotwein wurde leer (Grass)

(70) in einem Liter heißem Wasser (Konsalik)

(71) der Geruch eines Stücks silberbestickten Brokats (Süskind)

Der Anteil von Typ (iii) ist gegenüber der ersten Hälfte des Jahrhunderts wieder leicht angewachsen. Allerdings können die meisten dieser Belege alternativ als Typ (ii) gedeutet werden. Nur sechs Belege mit Erstglied im Dativ und maskulinem oder neutralem Zweitglied gehören eindeutig zu Typ (iii):

(72) weil sie ihm außer einer Flasche jugoslawischen Rotwein ... nur Coca-Cola anbieten konnte (Elsner)

(73) und griff nach einem Würfel roten Wachs (Heiduczek)

Die wenigen Belege vom Typ (iv) können wiederum alternativ als Typ (ii) oder (iii) analysiert werden. Typ (v) bleibt mit nur einem Prozent der Belege marginal. Die Erstglieder stehen im Akkusativ oder Dativ. Das Zweitglied ist stets ein Neutrum, in vier von fünf Belegen die lexikalisierte Verbindung Kölnisch Wasser.

Die Belege mit Erstglied im Akkusativ sind in der zweiten Hälfte des 20. Jhds. beim Genitivanschluss mit $60 \%$ gegenüber $65 \%$ immer noch unter- und bei den Konstruktionen mit Kasusübereinstimmung mit 68\% leicht überrepräsentiert. Gruppen mit Erstglied im Nominativ zeigen eine Präferenz für Konstruktionstyp (ii); nur solche mit Erstglied im Dativ bevorzugen weiterhin den Genitivanschluss.

Die Bevorzugung von Kasusübereinstimmung gegenüber Genitivanschluss bei maskulinen und neutralen Zweitgliedern (66\% gegenüber 34\% bei den Maskulina; $61 \%$ gegenüber $36 \%$ bei den Neutra) hat sich gegenüber der ersten Hälfte des 20. Jhds. leicht verstärkt. Bei den femininen Zweitgliedern ist der Anteil des Genitivanschlusses noch deutlich höher, aber auch hier macht er mit $45 \%$ jetzt weniger als die Hälfte der Belege aus. Wie schon in den vorherigen Untersuchungszeiträumen ist in den Gruppen mit singularischem Zweitglied der Genitivanschluss deutlich seltener als in Gruppen mit pluralischem Zweitglied. Kasusübereinstimmung ist deutlich stärker vertreten.

In der Gesamtentwicklung zeigt sich bei den Nquant1a-Gruppen eine fortschreitende Vereinheitlichung der Konstruktionen. Konstruktionstyp (v) (mit nicht-dekliniertem Adjektiv), der im 17. Jhd. den größten Anteil hatte, geht zugunsten des Genitivanschlusses stark zurück und bleibt nur als marginale Variante erhalten. Konstruktionstyp (i) war im 17. Jhd. auf Gruppen mit akkusativischem oder dativischem Erst- und neutralem oder femininem Zweitglied spezialisiert. Im 18. Jhd. dringt er auch in Gruppen mit nominativischem Erstglied und maskulinem Zweitglied vor. Konstruktionstyp (ii) kommt bis ins 19. Jhd. fast ausschließlich in Gruppen mit akkusativischem oder nominativischem Erstglied vor. In Gruppen mit dativischem Erstglied erscheint er erst im 20. Jahrhundert. Die Unterschiede in den Konstruktionspräferenzen zwischen Gruppen mit nominativischem vs. dativischem Erstglied und maskulinem vs. neutralem Zweitglied gehen sukzessive zurück. In allen Teilgruppen findet eine Konzentration auf die Alternative zwischen Genitivanschluss und Kasusübereinstimmung statt. 
Der in den Grammatikerurteilen aus dem 19. und 20. Jhd. beschriebene Rückgang des Genitivanschlusses zugunsten von Kasusübereinstimmung ist im Nquant1a-Korpus gut erkennbar. Allerdings war Genitivanschluss nach Nquantla im 17. und 18. Jhd. seltener als Kasusübereinstimmung. Im 20. Jhd. ist er wiederum seltener. Die Bevorzugung für den Genitivanschluss ist bei diesen Gruppen somit im Wesentlichen eine Besonderheit des 19. Jhds. Nur in zwei Teilgruppen fällt sie etwas deutlicher aus und zeigt sich vom 18. bis ins 20. Jhd.: nach Erstgliedern im Dativ und bei Zweitgliedern im Femininum.

In den folgenden Unterkapiteln werden nun Gruppen mit pluralischem Zweitglied genauer untersucht.

\subsection{Konstruktionen mit Einheitennomina und Zweitglied im Plural (Nquant1b)}

Nquant1b-Gruppen haben mit 518 Belegen einen Anteil von 20\% im Korpus. Formal sind sie weniger diversifiziert als Nquantla-Gruppen. Konstruktionstyp (v) fehlt bei ihnen völlig; Typ (iii) ist durchweg sehr schwach vertreten. Die Erstglieder der Nquant1b-Gruppen stehen zu mehr als 50\% im Akkusativ. Die Zweitglieder bezeichnen überwiegend Mengen unbelebter Entitäten. Deshalb fungieren diese Gruppen syntaktisch ebenfalls bevorzugt als Objekte.

\subsubsection{Jahrhundert}

Aus dem 17. Jhd. enthält das Korpus nur 24 Belege mit Nquant1b. Bei einer so kleinen Zahl müssen die quantitativen Verteilungen mit großer Vorsicht betrachtet werden. Bei der Hälfte der Belege steht das Erstglied im Akkusativ, bei einem guten Viertel im Nominativ, bei einem knappen Viertel im Dativ. Belege mit Erstglied im Genitiv fehlen:

\begin{tabular}{|l|c|c|c|c|}
\hline Erstglied & Nominativ & Akkusativ & Dativ & Summe \\
\hline Typ (i) & 3 & 5 & 3 & $11(46 \%)$ \\
\hline Typ (ii) & 2 & 3,5 & 1 & $6,5(26 \%)$ \\
\hline Typ (iii) & - & - & 1 & $1(4 \%)$ \\
\hline Typ (iv) & 2 & 3,5 & - & $5,5(24 \%)$ \\
\hline Summe & $7(29 \%)$ & $12(50 \%)$ & $5(21 \%)$ & $24(100 \%)$ \\
\hline
\end{tabular}

Tab. 13: Verteilung der Belege mit Nquant1b aus dem 17. Jhd.

Belege mit Genitivanschluss bilden knapp die Hälfte der Vorkommen. Damit haben sie einen mehr als doppelt so großen Anteil wie bei den Nquant1a-Gruppen:

(74) wiewol eine große Pfann gebackener Eier dort stunde (Grimmelshausen)

Die Konstruktionstypen (ii) und (iv) stellen jeweils etwa ein Viertel:

(75) mit einem Haufen jungen Hunden (Grimmelshausen)

(76) bekamen wir eine grosse menge kleine Fische (Olearius)

Auch der Anteil von Typ (ii) ist größer als bei den Nquant1a-Gruppen. Am deutlichsten ist der Unterschied aber bei Typ (iv) (weder Genitiv noch Kasusübereinstimmung), der bei den Nquant1a-Gruppen durchweg marginal ist.

Bei den Kasus der Erstglieder sind im Korpus zum 17. Jhd. keine Konstruktionspräferenzen erkennbar. 


\subsubsection{Jahrhundert}

Die Zahl der Belege aus dem 18. Jhd. ist größer, aber mit 41 immer noch sehr klein. Bei gut der Hälfte von ihnen steht das Erstglied im Akkusativ, bei jeweils knapp einem Viertel im Nominativ bzw. Dativ. Belege mit Erstglied im Genitiv fehlen:

\begin{tabular}{|l|c|c|c|c|}
\hline Erstglied & Nominativ & Akkusativ & Dativ & Summe \\
\hline Typ (i) & 8 & 20 & 9 & $37(90 \%)$ \\
\hline Typ (ii) & 0,5 & 1 & 0,5 & $2(5 \%)$ \\
\hline Typ (iii) & - & - & 0,5 & $0,5(1 \%)$ \\
\hline Typ (iv) & 0,5 & 1 & - & $1,5(4 \%)$ \\
\hline Summe & $9(22 \%)$ & $22(54 \%)$ & $10(24 \%)$ & $41(100 \%)$ \\
\hline
\end{tabular}

Tab. 14: Verteilung der Belege mit Nquant1b aus dem 18. Jhd.

Typ (i) stellt in dieser Beleggruppe 90\% der Vorkommen. Sein Anteil bleibt über den gesamten weiteren Untersuchungszeitraum stabil:

(77) Sie behandelten es als einen Haufen belebter Maschinen (Wieland)

(78) Große Haufen vergilbter alter Briefschaften lagen vor ihm (Goethe)

(79) Die ganze Ladung bestand aus sechs Ballen feiner Tücher (Campe)

Nur vier Belege sind den Typen (ii), (iii) und/oder (iv) zuzuordnen, darunter (80), der alternativ als Typ (ii) oder (iii) gedeutet werden kann:

(80) und ist der Leithammel von einer Menge jungen Leuten (Heinse)

\subsubsection{9. und 20. Jahrhundert}

Aus dem 19. Jhd. enthält das Korpus 90 Belege mit Nquant1b. Das Erstglied steht in 57\% im Akkusativ, in 32\% im Nominativ, in 11\% im Dativ. Wiederum fehlen Belege mit Erstglied im Genitiv:

\begin{tabular}{|l|c|c|c|c|}
\hline Erstglied & Nominativ & Akkusativ & Dativ & Summe \\
\hline Typ (i) & 26 & 46 & 9 & $81(90 \%)$ \\
\hline Typ (ii) & 1,5 & 2,5 & 0,5 & $4,5(5 \%)$ \\
\hline Typ (iii) & - & - & 0,5 & $0,5(0,5 \%)$ \\
\hline Typ (iv) & 1,5 & 2,5 & - & $4(4,5 \%)$ \\
\hline Summe & $29(32 \%)$ & $51(57 \%)$ & $10(11 \%)$ & $90(100 \%)$ \\
\hline
\end{tabular}

Tab. 15: Verteilung der Belege mit Nquant1b aus dem 19. Jhd.

Die Verteilung der Belege auf die Konstruktionstypen ist identisch wie im 18. Jhd. Eine unterschiedliche Verteilung bei den Kasus des Erstglieds ist nicht erkennbar. (81) illustriert Konstruktionstyp (i); (82) kann Typ (ii) oder (iv) zugeordnet werden:

(81) und welch eine Menge kleiner Tiere lief darin herum (Raabe)

(82) und nahm ein Schächtelchen isländische Moospastillen mit sich (Fontane)

Aus der ersten Hälfte des 20. Jhds. enthält das Korpus 150 Belege mit Nquant1b. Bei 58\% steht das Erstglied im Akkusativ, bei 32\% im Nominativ, bei 9\% im Dativ, bei 1\% im Genitiv: 


\begin{tabular}{|l|c|c|c|c|c|}
\hline Erstglied & Nominativ & Akkusativ & Dativ & Genitiv & Summe \\
\hline Typ (i) & 44 & 77 & 14 & 1 & $136(90 \%)$ \\
\hline Typ (ii) & 2 & 4,5 & - & 1 & $7,5(5 \%)$ \\
\hline Typ (iv) & 2 & 4,5 & - & - & $6,5(5 \%)$ \\
\hline Summe & $48(32 \%)$ & $86(58 \%)$ & $14(9 \%)$ & $2(1 \%)$ & $150(100 \%)$ \\
\hline
\end{tabular}

Tab. 16: Verteilung der Belege mit Nquant1b aus der ersten Hälfte des 20. Jhds.

In dieser Beleggruppe fehlt Konstruktionstyp (iii). Ansonsten ist die Verteilung identisch wie im 18. und 19. Jhd. Je ein Beleg für Typ (i) und (ii) bzw. (iv):

... weil er zu einem Haufen streikender Arbeiter sprach (Kellermann)

... und aß bedächtig eine Portion gebackene kleine Fische (Frank)

Auch in der zweiten Hälfte des 20. Jhds. bleibt die Verteilung konstant. Aus diesem Zeitraum enthält das Korpus 213 Belege. Bei 57\% steht das Erstglied im Akkusativ, bei 28\% im Nominativ, bei $15 \%$ im Dativ:

\begin{tabular}{|l|c|c|c|c|}
\hline Erstglied & Nominativ & Akkusativ & Dativ & Summe \\
\hline Typ (i) & 55 & 105 & 32 & $192(90 \%)$ \\
\hline Typ (ii) & 2 & 8 & 0,5 & $10,5(5 \%)$ \\
\hline Typ (iii) & - & - & 0,5 & $0,5(0 \%)$ \\
\hline Typ (iv) & 2 & 8 & - & $10(5 \%)$ \\
\hline Summe & $59(28 \%)$ & $121(57 \%)$ & $33(15 \%)$ & $213(100 \%)$ \\
\hline
\end{tabular}

Tab. 17: Verteilung der Belege mit Nquant1b aus der zweiten Hälfte des 20. Jhds.

Je ein Beleg vom Typ (i) und (ii) bzw. (iv):

(85) Bei einer Party machte sie sogar mit einer Dose ostafrikanischer Heuschrecken von sich reden (Elsner)

(86) Jochen verlangte sechs Bockwürste und eine Tüte saure Gurken (Morgner)

Nquant1b-Gruppen verhalten sich in zweierlei Hinsicht völlig anders als Nquant1a-Gruppen. Zum einen ist bei ihnen im Untersuchungszeitraum keine Entwicklung im engeren Sinne nachvollziehbar. Die Daten zeigen im 18. Jhd. eine Verteilung, die gegenüber dem 17. Jhd. sprunghaft und relativ stark verändert erscheint. Ab da bleibt sie bis zum Ende des Untersuchungszeitraums konstant. Zum anderen ist der Genitivanschluss in diesem Teilkorpus ab dem 18. Jhd. konkurrenzlos dominant. Kasusübereinstimmung und Konstruktionen, die weder Genitiv noch Kasusübereinstimmung aufweisen, waren nur im 17. Jhd. Alternativen mit nennenswerten Vorkommensanteilen. Ab dem 18. Jhd. spielen sie eine untergeordnete Rolle.

Wie bei den Nquantla-Gruppen zeigt sich auch bei den Nquant1b-Gruppen eine Vereinheitlichung der Konstruktionsweise. Diese findet innerhalb des Untersuchungszeitraums aber nicht in mehreren Schritten statt, sondern in nur einem Schritt vom 17. zum 18. Jhd., und sie führt nicht zu zwei etwa gleichberechtigten Konstruktionsalternativen, sondern zu einer eindeutig präferierten Konstruktion. Bei den Nquant1b-Gruppen ist weder eine weitere Zunahme des Genitivanschlusses vom 18. zum 19. Jhd. noch ein Rückgang des Genitivs im 20 Jhd. zu sehen. Die in Kapitel 2 referierten Grammatikautoren scheinen Nquant1b-Gruppen bei ihren Urteilen nicht im Blick gehabt zu haben. 
4.3 Konstruktionen mit Sammelbezeichnungen im engeren Sinne (Nquant2)

Nquant2-Gruppen haben mit 305 Belegen den kleinsten Anteil (12\%) im Korpus. Wie bei Nquant1b-Gruppen steht ihr Zweitglied im Plural. Formal sind sie im gesamten Untersuchungszeitraum kaum diversifiziert. Konstruktionstyp (i) ist durchweg stark dominant. Daneben kommen wenige Einzelbelege vor, die alternativ als Typ (ii) oder (iv) analysiert werden können. Die Typen (iii) und (v) fehlen völlig. Die Erstglieder von Nquant2-Gruppen stehen zu etwa einem Drittel im Nominativ und zu weniger als der Hälfte im Akkusativ. Das Zweitglied bezeichnet oft Personen; entsprechend kommen diese Gruppen häufiger als Subjekt vor.

Aus dem 17. Jhd. enthält das Korpus 10 Belege. Acht von ihnen haben ein Erstglied im Nominativ, zwei ein Erstglied im Dativ. Belege mit akkusativischem oder genitivischem Erstglied fehlen:

\begin{tabular}{|l|c|c|c|}
\hline Erstglied & Nominativ & Dativ & Summe \\
\hline Typ (i) & 6 & 2 & $8(80 \%)$ \\
\hline Typ (ii) & 1 & - & $1(10 \%)$ \\
\hline Typ (iv) & 1 & - & $1(10 \%)$ \\
\hline Summe & $8(80 \%)$ & $2(20 \%)$ & $10(100 \%)$ \\
\hline
\end{tabular}

Tab. 18: Verteilung der Belege mit Nquant2 aus dem 17. Jhd.

Die absolute Zahl der Belege ist für eine verlässliche quantitative Analyse zu klein. Deutlich ist aber, dass der Genitivanschluss nach Nquant2 bereits im 17. Jhd. der präferierte Konstruktionstyp ist. Bei dativischem Erstglied ist er möglicherweise alternativlos. Mit nominativischem Erstglied kommen Belege vor, die den Konstruktionstypen (ii) oder (iv) zugeordnet werden können:

(87) daß ich ihn bey einer lustigen Gesellschaft junger Kauffleute antraff (Grimmelshausen)

(88) etliche starke Troupen wol ausgeputzte Reuter (Olearius)

An dieser Verteilung ändert sich über den gesamten Untersuchungszeitraum hinweg nichts. Die anteilige Dominanz von Typ (i) wird nur noch deutlicher.

Aus dem 18. Jhd. enthält das Korpus 23 Belege. 57\% haben ein Erstglied im Akkusativ, $30 \%$ ein Erstglied im Nominativ, 13\% ein Erstglied im Dativ. Belege mit genitivischem Erstglied fehlen:

\begin{tabular}{|l|c|c|c|c|}
\hline Erstglied & Nominativ & Akkusativ & Dativ & Summe \\
\hline Typ (i) & 5 & 13 & 3 & $21(91 \%)$ \\
\hline Typ (ii) & 1 & - & - & $1(4,5 \%)$ \\
\hline Typ (iv) & 1 & - & - & $1(4,5 \%)$ \\
\hline Summe & $7(30 \%)$ & $13(57 \%)$ & $3(13 \%)$ & $23(100 \%)$ \\
\hline
\end{tabular}

Tab. 19: Verteilung der Belege mit Nquant2 aus dem 18. Jhd.

Je ein Beleg für die Konstruktionstypen (i) und (ii)/(iv):

(89) Man erblickte einen Trupp bewaffneter Männer ... (Goethe)

(90) ... gehen eine Anzahl mit einem besondern Schilde bezeichnete Personen herum (Nikolai) 
Aus dem 19. Jhd. enthält das Korpus 105 Belege. 43\% haben ein Erstglied im Akkusativ, 36\% ein Erstglied im Nominativ, 21\% ein Erstglied im Dativ:

\begin{tabular}{|l|c|c|c|c|}
\hline Erstglied & Nominativ & Akkusativ & Dativ & Summe \\
\hline Typ (i) & 37 & 45 & 22 & $104(99 \%)$ \\
\hline Typ (ii) & 0,5 & - & - & $0,5(0,5 \%)$ \\
\hline Typ (iv) & 0,5 & - & - & $0,5(0,5 \%)$ \\
\hline Summe & $38(36 \%)$ & $45(43 \%)$ & $22(21 \%)$ & $105(100 \%)$ \\
\hline
\end{tabular}

Tab. 20: Verteilung der Belege mit Nquant2 aus dem 19. Jhd.

Je ein Beispiel für Typ (i) und Typ (ii)/(iv):

(91) Ich brachte eine ganze Reihe ähnlicher Fragen dadurch zum Abschluß ... (Raabe)

(92) worauf drei Reihen eiserne Töpfe standen (Heine)

Aus der ersten Hälfte des 20. Jhds. enthält das Korpus 77 Belege. 52\% haben ein Erstglied im Akkusativ, 26\% ein Erstglied im Nominativ, 22\% ein Erstglied im Dativ. Sämtliche Belege haben Genitivanschluss:

\begin{tabular}{|l|c|c|c|c|}
\hline Erstglied & Nominativ & Akkusativ & Dativ & Summe \\
\hline Typ (i) & 20 & 40 & 17 & $77(100 \%)$ \\
\hline Summe & $20(26 \%)$ & $40(52 \%)$ & $17(22 \%)$ & $77(100 \%)$ \\
\hline
\end{tabular}

Tab. 21: Verteilung der Belege mit Nquant2 aus der ersten Hälfte des 20. Jhds.

Aus der zweiten Hälfte des 20. Jhds. enthält das Korpus 90 Belege. 43\% haben ein Erstglied im Nominativ, 37\% ein Erstglied im Akkusativ, 17\% ein Erstglied im Dativ und 1\% ein Erstglied im Genitiv. Auch hier dominiert Konstruktionstyp (i) fast vollständig:

\begin{tabular}{|l|c|c|c|c|c|}
\hline Erstglied & Nominativ & Akkusativ & Dativ & Genitiv & Summe \\
\hline Typ (i) & 36 & 33 & 17 & 0,5 & $86,5(96 \%)$ \\
\hline Typ (ii) & 1,5 & - & - & 0,5 & $2(2 \%)$ \\
\hline Typ (iv) & 1,5 & - & - & - & $1,5(2 \%)$ \\
\hline Summe & $39(43 \%)$ & $33(37 \%)$ & $17(19 \%)$ & $1(1 \%)$ & $90(100 \%)$ \\
\hline
\end{tabular}

Tab. 22: Verteilung der Belege mit Nquant2 aus der zweiten Hälfte des 20. Jhds.

Die Datenlage in den Nquant2-Gruppen widerspricht den in Kapitel 2 referierten Grammatikerurteilen am deutlichsten. Genitivanschluss ist im gesamten Untersuchungszeitraum der dominante Konstruktionstyp. Zumindest in der relativ kleinen Belegzahl des Korpus ist vom 19. zum 20. Jhd. keine relevante Veränderung zu beobachten.

\subsection{Konstruktionen mit Zahlnomina (Nquant3)}

Nquant3-Gruppen haben mit 574 Belegen einen Anteil von 22\% am Korpus. Ihr Zweitglied steht im Plural. Sie zeigen aber eine weitaus größere konstruktionelle Varianz als andere Gruppen mit pluralischem Zweitglied. Über den gesamten Untersuchungszeitraum hinweg sind die Konstruktionstypen (i), (ii) und (iv) annähernd gleichgewichtige Alternativen. Im 17. Jhd. sind die Typen (iii) und (v) ebenfalls noch belegt. Typ (v) verschwindet danach. Typ (iii) wird allmählich seltener und verschwindet in der zweiten Hälfte des 20. Jhds. Mehr als die Hälfte der Erstglieder der Nquant3-Gruppen steht im Akkusativ. 


\subsubsection{Jahrhundert}

Aus dem 17. Jhd. enthält das Korpus 34 Belege. Ihr Erstglied steht zu 70\% im Akkusativ, zu 21\% im Nominativ und zu 9\% im Dativ. Belege mit genitivischem Erstglied fehlen:

\begin{tabular}{|l|c|c|c|c|}
\hline Erstglied & Nominativ & Akkusativ & Dativ & Summe \\
\hline Typ (i) & 3 & 7 & 1 & $11(32 \%)$ \\
\hline Typ (ii) & 2 & 7,5 & 1 & $10,5(31 \%)$ \\
\hline Typ (iii) & - & - & 1 & $1(3 \%)$ \\
\hline Typ (iv) & 2 & 7,5 & - & $9,5(28 \%)$ \\
\hline Typ (v) & - & 2 & - & $2(6 \%)$ \\
\hline Summe & $7(21 \%)$ & $24(70 \%)$ & $3(9 \%)$ & $34(100 \%)$ \\
\hline
\end{tabular}

Tab. 23: Verteilung der Belege mit Nquant3 aus dem 17. Jhd.

Konstruktionen vom Typ (i) bilden ein knappes Drittel der Gesamtvorkommen:

(93) und er hatte nicht nur ein halb dutzet rechtschaffener und wol-qualificirter Auffwarter (Grimmelshausen)

Die meisten Belege ohne Genitivanschluss mit Erstglied im Nominativ oder Akkusativ können alternativ Typ (ii) oder (iv) zugeordnet werden:

(94) daß viel Leute ein halb Schock kleine und unnütze Stubenklecker halten (Weise)

Interessant ist ein Beleg, in dem Genitivanschluss (Typ i) und Kasusübereinstimmung (Typ ii) in der gleichen Nominalgruppe direkt nebeneinanderstehen:

(95) ich fande ... kein eintziges paar neuer, weder kleine noch große Schuh (Grimmelshausen)

Typ (v) kommt nur mit Erstglied im Akkusativ vor:

(96) verehrte er der Corinne ... ein stück Atlas, ein paar Seiden Strümpff und zehen Stück Band (Schupp)

Nach dem Kasus des Erstglieds zeigen sich in dieser Beleggruppe keine Präferenzen für einzelne Konstruktionstypen. Durchweg hat etwa ein Drittel der Belege Genitivanschluss. Zwei Drittel sind ohne Genitiv konstruiert; die meisten dieser Belege erlauben die Deutung, dass Erst- und Zweitglied im Kasus übereinstimmen.

Die Verteilung der Konstruktionstypen nach Nquant3 ist der nach Nquant1a/b ähnlich. Genitivanschluss ist nach Nquant3 seltener als sonst bei pluralischem Zweitglied. Als etwa gleich starke Konstruktionsalternativen stehen wie nach Nquant1b die Typen (ii) und (iv) zur Verfügung.

\subsubsection{Jahrhundert}

Aus dem 18. Jhd. enthält das Korpus 79 Belege. Bei 61\% steht das Erstglied im Akkusativ, bei 20\% im Nominativ, bei 16\% im Dativ, bei 3\% im Genitiv:

\begin{tabular}{|l|c|c|c|c|c|}
\hline Erstglied & Nominativ & Akkusativ & Dativ & Genitiv & Summe \\
\hline Typ (i) & 4 & 8 & 6 & 1 & $19(25 \%)$ \\
\hline Typ (ii) & 6 & 20 & 3,5 & 1 & $30,5(38 \%)$ \\
\hline Typ (iii) & - & - & 3,5 & - & $3,5(4,5 \%)$ \\
\hline Typ (iv) & 6 & 20 & - & & $26(32,5 \%)$ \\
\hline Summe & $16(20 \%)$ & $48(61 \%)$ & $13(16 \%)$ & $2(3 \%)$ & $79(100 \%)$ \\
\hline
\end{tabular}

Tab. 24: Verteilung der Belege mit Nquant3 aus dem 18. Jhd. 
Genitivanschluss ist auf ein Viertel der Belege zurückgegangen. Belege mit Erstglied im Dativ sind dabei über-, solche mit Erstglied im Akkusativ unterrepräsentiert:

(97) von Millionen künstiger Seelen (Heinse)

Die übrigen Belege sind als Typ (ii) oder (iv) deutbar, wenn das Erstglied im Nominativ oder Akkusativ steht:

(98) Wie ein Paar junge Götter lagen wir da im Schatten (Heinse)

Steht das Erstglied im Dativ, so kann der Beleg Typ (ii) oder (iii) zugeordnet werden:

(99) mit einem Paar kleinen Augen (Wieland)

Gegenüber dem 17. Jhd. sind Konstruktionen ohne Genitivanschluss auf 75\% angewachsen. Nach wie vor besteht eine recht deutliche Ähnlichkeit in der Verteilung der Konstruktionstypen zwischen Nquant3 und Nquant1a. Im 18. Jhd. ist der Genitivanschluss nach Nquant3 seltener als nach allen anderen Nquant-Klassen.

\subsubsection{Jahrhundert}

Aus dem 19. Jhd. enthält das Korpus 95 Belege. Bei 50\% steht das Erstglied im Akkusativ, bei $25 \%$ im Nominativ, bei $23 \%$ im Dativ, bei $2 \%$ im Genitiv:

\begin{tabular}{|l|c|c|c|c|c|}
\hline Erstglied & Nominativ & Akkusativ & Dativ & Genitiv & Summe \\
\hline Typ (i) & 13 & 7 & 11 & 1 & $32(34 \%)$ \\
\hline Typ (ii) & 5,5 & 20 & 5,5 & 1 & $32(34 \%)$ \\
\hline Typ (iii) & - & - & 5,5 & - & $5,5(6 \%)$ \\
\hline Typ (iv) & 5,5 & 20 & - & - & $25,5(26 \%)$ \\
\hline Summe & $24(25 \%)$ & $47(50 \%)$ & $22(23 \%)$ & $2(2 \%)$ & $95(100 \%)$ \\
\hline
\end{tabular}

Tab. 25: Verteilung der Belege mit Nquant3 aus dem 19. Jhd.

Der Anteil der Belege mit Genitivanschluss ist wieder auf gut ein Drittel gestiegen:

(100) sah aber nichts als ein Paar glühroter Augen (Immermann)

Die übrigen Belege können nach Erstglied im Nominativ oder Akkusativ den Typen (ii) oder (iv), nach Erstglied im Dativ den Typen (ii) oder (iii) zugeordnet werden:

(101) zu denen sich bald zwei Dutzend alte Weiber gesellten (Heine)

(102) mit einem Paar blauen Augen (Storm)

Beim Genitivanschluss sind Belege mit Erstglied im Nominativ und Dativ anteilig über-, solche mit Erstglied im Akkusativ unterrepräsentiert. Ähnliche Beobachtungen hatten wir bei den Nquantla-Gruppen gemacht. Auch im 19. Jhd. bleibt der Genitivanschluss nach Nquant3 seltener als nach allen anderen Nquant-Klassen.

\subsubsection{Erste Hälfte des 20. Jahrhunderts}

Aus der ersten Hälfte des 20. Jhds. enthält das Korpus 220 Belege. Bei 55\% steht das Erstglied im Akkusativ, bei 31\% im Nominativ, bei 13\% im Dativ, bei 1\% im Genitiv: 


\begin{tabular}{|l|c|c|c|c|c|}
\hline Erstglied & Nominativ & Akkusativ & Dativ & Genitiv & Summe \\
\hline Typ (i) & 43 & 49 & 27 & 1 & $120(54,5 \%)$ \\
\hline Typ (ii) & 12,5 & 36 & 1 & 1 & $50,5(23 \%)$ \\
\hline Typ (iii) & - & - & 1 & - & $1(0,5 \%)$ \\
\hline Typ (iv) & 12,5 & 36 & - & - & $48,5(22 \%)$ \\
\hline Summe & $68(31 \%)$ & $121(55 \%)$ & $29(13 \%)$ & $2(1 \%)$ & $220(100 \%)$ \\
\hline
\end{tabular}

Tab. 26: Verteilung der Belege mit Nquant3 aus der ersten Hälfte des 20. Jhds.

Anders als nach den übrigen Nquant-Klassen nimmt der Anteil des Genitivanschlusses nach Nquant3 im 20. Jhd. kräftig zu. In der ersten Jahrhunderthälfte macht er mehr als die Hälfte der Belege aus:

(103) Jeder Seemann kennt zwei Dutzend englischer Wörter (Traven)

Typ (ii), (iii) und (iv) gehen entsprechend zurück:

(104) Dafür hatte er sich ein Paar eiserne Hanteln angeschafft (Hesse)

(105) Sie sind die Meisterwerke von Millionen großen Chemikern (Kellermann)

Weiterhin ist der Genitivanschluss nach Erstgliedern im Akkusativ anteilig unter-, nach solchen im Nominativ und vor allem im Dativ überrepräsentiert.

\subsubsection{Zweite Hälfte des 20. Jahrhunderts}

Aus der zweiten Hälfte des 20. Jhds. enthält das Korpus 146 Belege. Bei 60\% steht das Erstglied im Akkusativ, bei 29\% im Nominativ, bei 11\% im Dativ. Belege mit genitivischem Erstglied fehlen:

\begin{tabular}{|l|c|c|c|c|}
\hline Erstglied & Nominativ & Akkusativ & Dativ & Summe \\
\hline Typ (i) & 21 & 29 & 16 & $66(45 \%)$ \\
\hline Typ (ii) & 11 & 29 & - & $40(27,5 \%)$ \\
\hline Typ (iv) & 11 & 29 & - & $40(27,5 \%)$ \\
\hline Summe & $43(29 \%)$ & $87(60 \%)$ & $16(11 \%)$ & $146(100 \%)$ \\
\hline
\end{tabular}

Tab. 27: Verteilung der Belege mit Nquant3 aus der zweiten Hälfte des 20. Jhds.

In der zweiten Jahrhunderthälfte geht der Genitivanschluss nach Nquant3, ähnlich wie nach Nquant1a, um etwa 10 Prozentpunkte zurück. Nach einem Erstglied im Akkusativ ist er anteilig weiterhin unterrepräsentiert. Nach einem Erstglied im Dativ folgt ausschließlich der Genitiv:

(106) Der Drack hätte ein Dutzend guter Partien machen können (Bernhard)

(107) Ein Paar roter Schuhe für Ann hängen ihm um den Hals (Wolf)

(108) ... blieb es einem Paar flacher, blauer Pumps überlassen (Konsalik)

Die Typen (ii) bzw. (iv) haben zusammen wieder einen Anteil von mehr als 50\%, sind aber ausschließlich nach Erstgliedern im Nominativ bzw. Akkusativ vertreten:

(109) ... hingen zwei Dutzend schmal schwarzgerahmte Fotos (Grass)

Auch nach Nquant3 zeigt sich die überall im Korpus beobachtete Tendenz zur Vereinheitlichung der Konstruktionen. Im 20. Jhd. sind Genitivanschluss und Kasusübereinstimmung die wichtigsten Konstruktionsalternativen mit einem Anteil von jeweils etwa 50\%. 
Damit ist der Genitivanschluss nach Nquant3 weitaus weniger prominent als sonst bei pluralischem Zweitglied, aber prominenter als bei singularischem Zweitglied. Die kräftige anteilige Zunahme des Genitivanschlusses, die bei den übrigen Nquant-Klassen vom 17. zum 18. bzw. vom 18. zum 19. Jhd. beobachtet wurde, findet nach Nquant3 erst vom 19. zum 20. Jhd. statt. In der zweiten Jahrhunderthälfte setzt ein leichter Rückgang des Genitivanschlusses ein, ähnlich wie sonst bei singularischem Zweitglied.

\section{Auswertung und Deutung}

Unsere Untersuchung hat ein detailliertes datengestütztes Bild der grammatischen Entwicklung von Nquant+Adj+N-Gruppen in der neuhochdeutschen Literatursprache ergeben. Die beschriebene Entwicklung unterscheidet sich deutlich von der bei Nquant+N-Gruppen ohne Adjektivattribut. Während bei letzteren der Genitivanschluss als Konstruktionstyp seit dem 16. Jhd. zurückgegangen und in der Gegenwartssprache so gut wie verschwunden ist, wuchs sein Anteil bei Nquant+Adj+N-Gruppen vom 17. bis zum 19. Jhd. stark an, wahrscheinlich mitbedingt durch Einflüsse normativer Grammatiken. Im 20. Jhd. zeigt sich ein leichter Rückgang, der aber viel schwächer ausfällt, als die Grammatiken der Gegenwartssprache es glauben machen.

Die Gesamtentwicklung der neuhochdeutschen Nquant+Adj+N-Gruppen ist durch eine konstruktionelle Vereinheitlichung gekennzeichnet. Im 17. Jhd. stehen fünf Konstruktionstypen als annähernd gleich starke Varianten nebeneinander. In unserem Korpus erscheint das Zweitglied in etwa einem Drittel der Belege als Erweiterung zum Erstglied (Typ i); in einem weiteren Drittel kann das Erstglied als Erweiterung zum Zweitglied gedeutet werden (Typen ii und iii); im dritten Drittel bleibt die syntaktische Binnenstruktur der Nquant+Adj+N-Gruppe unbestimmt (Typen iv und v). Im 18. und 19. Jhd. setzt der Genitivanschluss (Typ i) sich als dominante Konstruktion durch. Kasusübereinstimmung (vor allem Typ ii) bleibt als Alternative annähernd stabil. Die Konstruktionstypen (iii), (iv) und (v) verschwinden nicht völlig, treten aber stark in den Hintergrund. Im 20. Jhd. geht Typ (i) leicht zugunsten von Typ (ii) zurück, bleibt aber weiterhin eindeutig dominant:

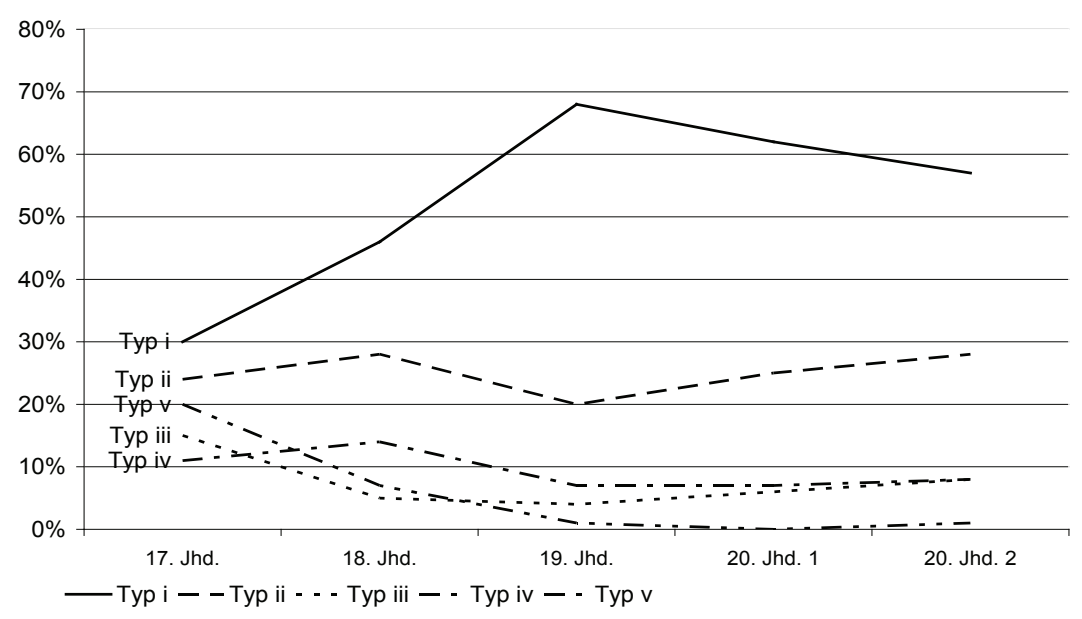

Abb. 1: Anteilige Entwicklung der Konstruktionstypen im Korpus 
Die Verteilung der Konstruktionstypen wird neben den vermuteten Einflüssen sprachexterner Normen erkennbar durch sprachinterne, grammatische Faktoren beeinflusst: die semantische Klasse und den Kasus des Erstglieds sowie Genus bzw. Numerus des Zweitglieds. Die Rolle der semantischen Klasse des Erstglieds ist in Kapitel 4 hinreichend klar geworden. Bezüglich Kasus des Erstglieds und Genus bzw. Numerus des Zweitglieds lässt unsere Untersuchung drei Tendenzen erkennen, die über den gesamten Untersuchungszeitraum hinweg sichtbar sind und auch in der Gegenwartssprache die Konstruktionswahl mitbestimmen (vgl. teilweise ähnliche Beobachtungen in Duden 2001, S. 590 und Duden 2009, S. 984ff. sowie bei Blatz 1896, S. 376):

1. Erstglieder im Dativ bevorzugen Genitivanschluss. Nach Erstgliedern im Akkusativ ist er weniger bevorzugt.

2. Pluralische und feminine Zweitglieder bevorzugen Genitivanschluss. Vor neutralen Zweitgliedern ist er weniger bevorzugt.

3. Maskuline und neutrale Erstglieder im Dativ akzeptieren schwache Adjektivdeklination (Typ iii).

Tendenz 3 ist relativ leicht mit dem Prinzip der Monoflexion zu erklären, das für Nominalgruppen des Deutschen immer wieder postuliert worden ist (vgl. Duden 2009, S. 947). Der Dativ der Maskulina und Neutra ist durch das $m$-Suffix auffällig und gut erkennbar. So kann im Zweitglied bei Kasusübereinstimmung auf eine zweite starke Dativ-Markierung verzichtet werden. Weitaus häufiger ist dennoch die starke Flexion des Zweitglieds (Typ ii) und vor allem der Genitivanschluss (Typ i).

Die Tendenzen 1 und 2 verlangen aufwändigere Erklärungen. Zunächst zu Tendenz 1. Abbildung 2 zeigt die Entwicklung des Genitivanschlusses im Gesamtkorpus in Abhängigkeit vom Kasus des Erstglieds.

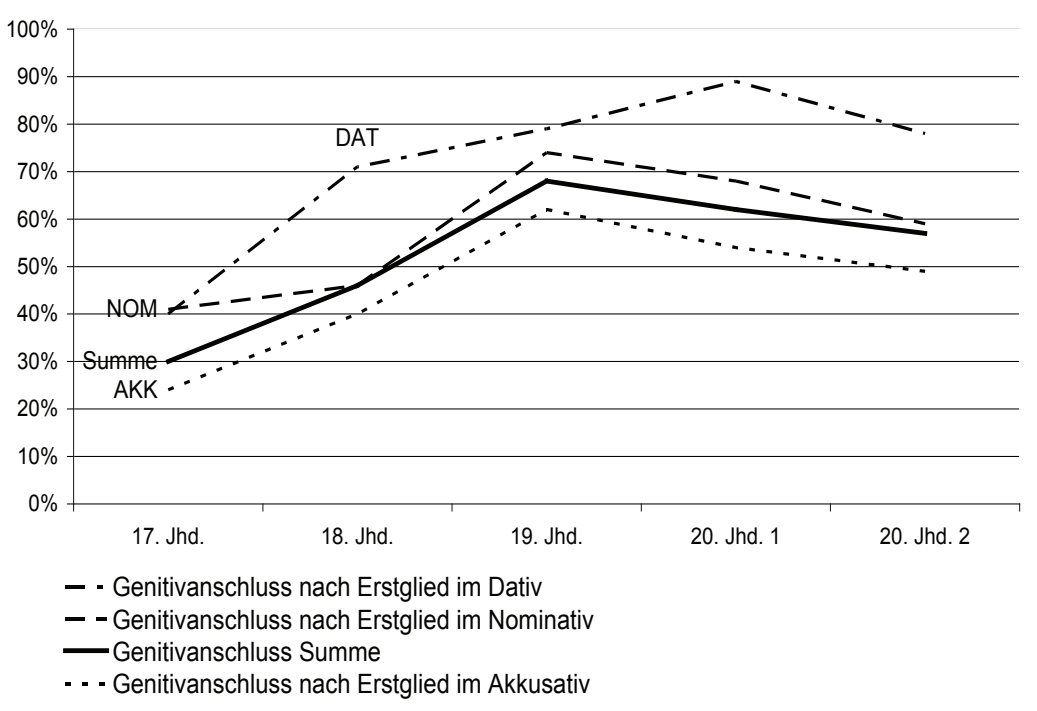

Abb. 2: Entwicklung des Genitivanschlusses in Abhängigkeit vom Kasus des Erstglieds

Warum ist Genitivanschluss nach Erstgliedern im Dativ deutlich stärker präferiert als nach Erstgliedern im Akkusativ? Nquant+Adj+N-Gruppen mit Erstglied im Dativ sind typischerweise Ergänzungen von Präpositionen. Die Präposition steht unmittelbar vor dem 
Erstglied und ordnet diesem auf kürzestmögliche syntaktische Distanz den Kasus zu. Diese Konfiguration begünstigt es, das Erstglied als Kern der Gruppe und das Zweitglied als Attribut zu deuten. Genitivanschluss ist eine Möglichkeit, den Attributstatus formal zum Ausdruck zu bringen.

Nquant+Adj+N-Gruppen mit Erstglied im Akkusativ sind dagegen typischerweise Ergänzungen zu Verben. Das Verb kann dem Erstglied unmittelbar vorausgehen, kann im Satz aber auch rechts der Nquant+Adj+N-Gruppe und/oder in Distanz zu ihr stehen. Wenn wir der Annahme der generativen Syntax folgen, dass Präpositionalphrasen im Deutschen linksköpfig, Verbalphrasen dagegen in der Grundstruktur rechtsköpfig sind (vgl. Lohnstein 2014, S. 168f.), so erhalten Nquant+Adj+N-Gruppen mit Erstglied im Akkusativ ihren Kasus typischerweise von rechts. Aus dieser Richtung trifft er zuerst auf das Zweitglied. Hinzu kommt, dass der von einem Verb regierte Akkusativ im Deutschen gewöhnlich mit der Zuordnung der thematischen Patiensrolle gekoppelt ist. Patiens zum Verb in einem Ausdruck wie ein Glas kaltes Wasser trinken kann sinnvollerweise nur das Zweitglied der Gruppe (hier: kaltes Wasser) sein, nur in Ausnahmefällen das quantifizierende Erstglied (hier: ein Glas). Beide Faktoren legen es nahe, in solchen Ausdrücken das Zweitglied als Kern der Konstruktion und das Erstglied als Erweiterung zu deuten. Dem entspricht stärker die Kasusübereinstimmung als Konstruktionstyp.

Nquant+Adj+N-Gruppen mit Erstglied im Nominativ zeigen ein uneinheitlicheres Verhalten. Im Gesamtkorpus liegt die Häufigkeit des Genitivanschlusses bei ihnen leicht über dem Durchschnitt. Betrachtet man aber nur die Belege mit singularischem Zweitglied, so ist sie überwiegend unterdurchschnittlich (vgl. Abb. 3):

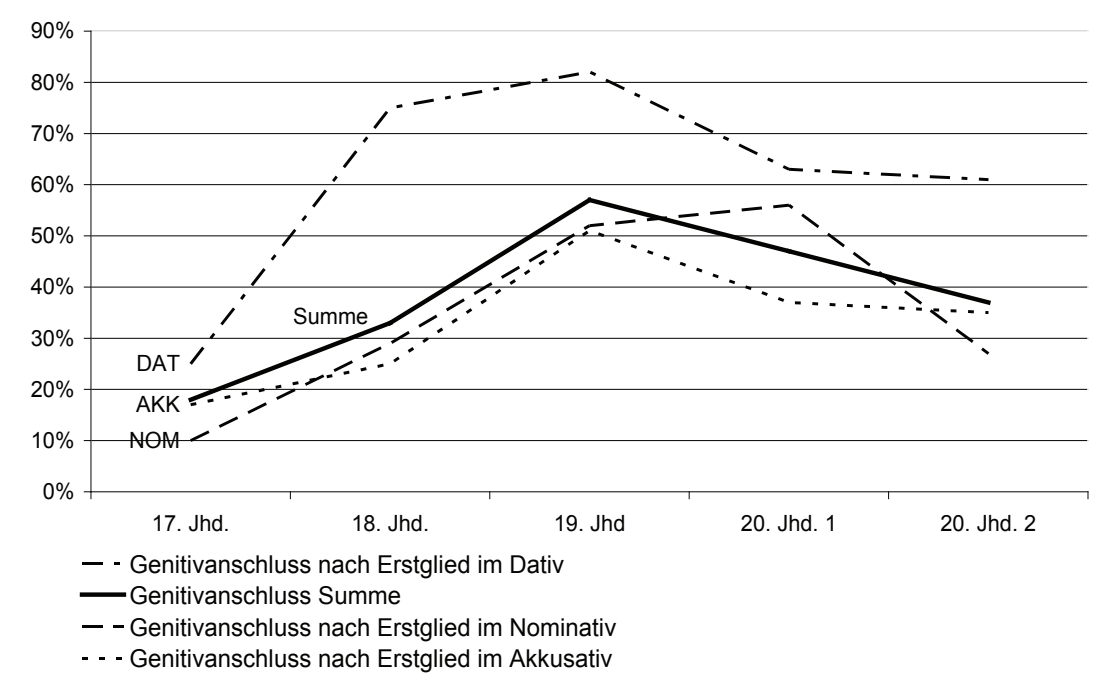

Abb. 3: Entwicklung des Genitivanschlusses vor singularischen Zweitgliedern

Vor singularischem Zweitglied ist der Genitivanschluss insgesamt weniger frequent als im Gesamtkorpus; der Verlauf der Durchschnittsskurve ist aber sehr ähnlich. Auch hier sieht man deutlich die Bevorzugung des Genitivanschlusses nach Erstgliedern im Dativ und seinen unterdurchschnittlichen Anteil nach Erstgliedern im Akkusativ. Die Kurve für den Genitivanschluss nach Erstgliedern im Nominativ ähnelt stark der Gesamtkurve, ist aber auf der Zeitachse nach rechts verschoben; in der zweiten Hälfte des 20. Jhds. geht die Frequenz des Genitivanschlusses überdurchschnittlich stark zurück. 
Nominalgruppen im Nominativ fungieren typischerweise als Subjekte. Ihre thematische Rolle erhalten sie ebenso wie Objekte vom Verb, dessen Grundposition in der Verbalphrase rechts von ihnen liegt. Wie sie ihren Kasus erhalten, wird von den Grammatiktheorien unterschiedlich erklärt. Im Satz sind Subjekte sehr viel lockerer als Objekte mit dem Verb verbunden und weitaus beweglicher als diese. Im Prinzip scheinen Subjekte, ähnlich wie Objekte, den Genitivanschluss nicht zu präferieren, aber aufgrund ihrer weniger engen syntaktischen Bindung ans Verb sind sie zugänglicher für weitere Einflussfaktoren, z.B. den Numerus des Zweitglieds oder auch sprachexterne Normen. So könnte es sich erklären, dass sich der von den Grammatikern geforderte Genitivanschluss bei ihnen mit leichter zeitlicher Verzögerung durchsetzt und, sobald die betreffenden Normen aufgegeben sind (in der zweiten Hälfte des 20. Jhds.), überdurchschnittlich stark zurückgeht.

Nun zu Tendenz 2: Warum ist Genitivanschluss gerade vor pluralischen und femininen Nomina im Zweitglied präferiert, bei denen der Genitiv im Neuhochdeutschen formal nicht ausgedrückt wird; warum ist er vor neutralen Nomina im Zweitglied, die den Genitiv formal anzeigen, weniger bevorzugt? Abbildung 4 gibt einen Überblick über die Entwicklung des Genitivanschlusses in Abhängigkeit vom Genus bzw. Numerus des Zweitglieds.

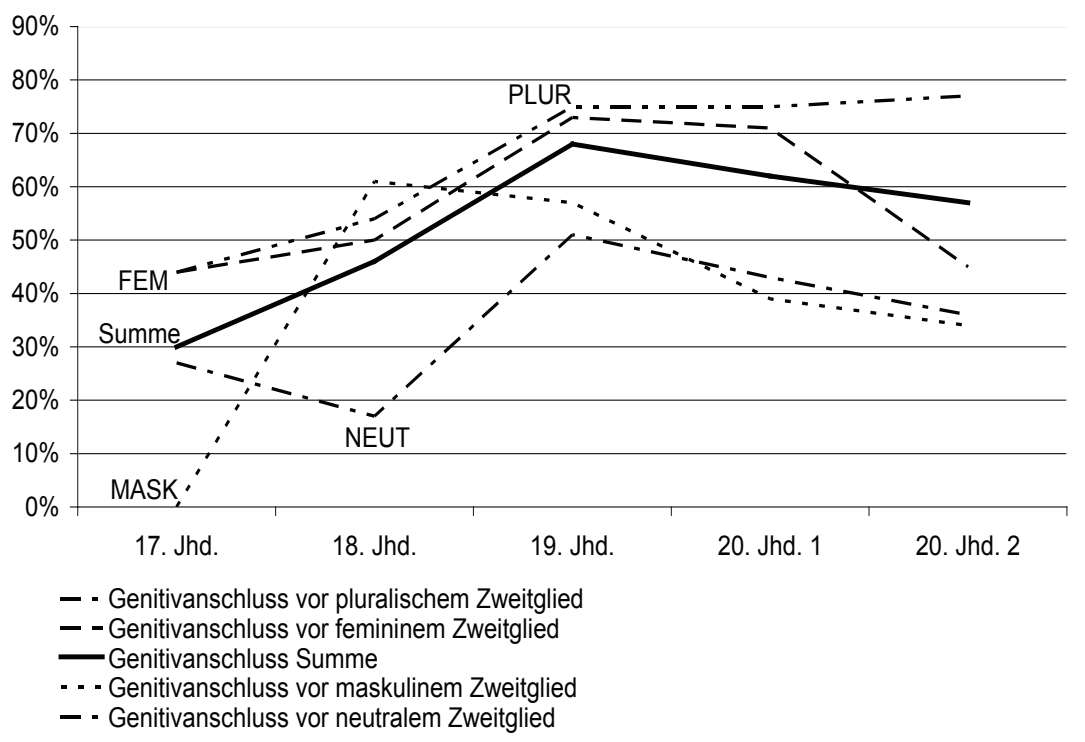

Abb. 4: Entwicklung des Genitivanschlusses in Abhängigkeit vom Genus bzw. Numerus des Zweitglieds

In Nquant $+\mathrm{N}-$ Gruppen ohne Adjektivattribut mit pluralischem oder femininem Zweitglied wie ein Glas Milch oder eine Handvoll Erbsen ist an der Form nicht erkennbar, ob das Zweitglied im Genitiv oder in einem anderen Kasus steht. In Nquant+Adj+N-Gruppen wie ein Glas frischer Milch oder eine Handvoll grüner Erbsen ist der Genitiv nur beim Adjektiv sichtbar. Beim Nomen des Zweitglieds besteht kein Formunterschied zwischen Nquant $+\mathrm{N}-$ Gruppen und Nquant+Adj+N-Gruppen, gleichgültig ob das Zweitglied im Genitiv steht oder nicht. Nominalgruppen wie ein Glas Milch und ein Glas frische(r) Milch bzw. eine Handvoll Erbsen und eine Handvoll grüne(r) Erbsen können problemlos der gleichen grammatischen Kategorie zugeordnet werden. Ein Genitivanschluss steht dem nicht im Weg. Dieser Umstand kann erklären, warum Nquant+Adj+N-Gruppen mit femininem oder plura- 
lischem Zweitglied einen von der Norm verlangten Genitivanschluss leicht annehmen und ihn nur relativ langsam abstoßen, wenn die Norm ihn nicht mehr verlangt.

Bei neutralen Nomina im Zweitglied ist die Lage anders. Sie haben eine eigene Genitivform, z.B. Wassers. In Nquant+N-Gruppen ohne Adjektivattribut ist der Genitivanschluss im Neuhochdeutschen nicht mehr produktiv: 'ein Schwall Wassers. Hier ist nur die endungslose Non-Genitiv-Form zugelassen: ein Schwall Wasser. In Nquant+Adj+NGruppen entspricht der Genitivanschluss aber bis ins 20. Jhd. hinein der Norm: ein Schwall schmutzigen Wassers. Allerdings hat der Formunterschied beim Nomen zwischen Nquant+N-Gruppen wie ein Schwall Wasser und Nquant+Adj+N-Gruppen wie ein Schwall schmutzigen Wassers zur Folge, dass sie weniger leicht als Instanzen der gleichen grammatischen Kategorie erkennbar sind. Der Bedarf, ihre Zuordnung zur gleichen Kategorie zu erleichtern, kann möglicherweise das seltenere Vorkommen des Genitivanschlusses vor neutralen Zweitgliedern erklären.

Bei maskulinen Nomina im Zweitglied ist die Lage ebenso. Nquant+N-Gruppen mit Genitiv wie ?eine Wolke Nebels sind im Neuhochdeutschen nicht mehr produktiv. Zwischen Nquant+N-Gruppen mit non-genitivischem Zweitglied wie eine Wolke Nebel und Nquant+Adj+N-Gruppen mit genitivischem Zweitglied wie eine Wolke dichten Nebels besteht ein Formunterschied, der es erschwert, beide als Instanzen der gleichen grammatischen Kategorie zu erkennen.

Wenn wir nun in Abbildung 4 die Kurve für die Nquant+Adj+N-Gruppen mit maskulinem Zweitglied betrachten, so sehen wir, dass auch sie überwiegend unterhalb der Durchschnittskurve liegt. Allerdings zeigt sie einen auffälligen Anstieg vom 17. zum 18. Jhd., der sie kurzzeitig über das Niveau aller übrigen Kurven hinaushebt. Unser Korpus enthält anteilig weniger Daten für diese beiden Jahrhunderte, sodass hier möglicherwiese ein statistisches Artefakt vorliegt. Allerdings sieht die Kurve für die Gruppen mit maskulinem Zweitglied in ihrem Gesamtverlauf der Durchschnittskurve sehr ähnlich, nur mit einer Verschiebung nach links auf der Zeitachse. Der anfängliche Anstieg verläuft wesentlich steiler als im Durchschnitt, erreicht nicht ganz dieselbe Höhe, und der Rückgang setzt deutlich früher ein. Das deutet darauf hin, dass Maskulina (als prototypische Nomina) Vorreiter der grammatischen Entwicklung sein könnten, während die Neutra eher nachfolgen. Dazu passt, dass die Grammatiker ihre Intuitionen über die Struktur von Nquant+Adj+N-Gruppen offenbar bevorzugt auf Gruppen mit maskulinem Zweitglied stützen. Zumindest wird das durch die Beispielwahl in der Grammatikliteratur nahegelegt (vgl. etwa die Zitate in Kap. 2).

Im Gesamtbild präzisieren die Befunde der vorliegenden Untersuchung einige schon bekannte Beobachtungen, widersprechen aber zum Teil auch deutlich den bisherigen Darstellungen. Relativierend ist nochmals darauf hinzuweisen, dass wir ausschließlich literatursprachliche Daten berücksichtigt haben. Unser Korpus enthält keine Daten aus der Alltagssprache, aus Fachsprachen oder aus anderen Funktionsbereichen. Dort kann die Entwicklung teilweise anders verlaufen sein. Insbesondere könnte in nicht-literarischen Sprachregistern im 20. Jhd. ein stärkerer Rückgang des Genitivanschlusses erfolgt sein, als wir ihn hier zeigen konnten. Das muss an weiteren Daten studiert werden. 


\section{Literatur}

Adelung, Johann Christoph (1782): Umständliches Lehrgebäude der deutschen Sprache zur Erläuterung der deutschen Sprachlehre für Schulen. 2 Bände. Leipzig: Breitkopf. [Nachdruck Hildesheim: Olms, 1971].

Andresen, Karl Gustav (1898): Sprachgebrauch und Sprachrichtigkeit im Deutschen. 8. Aufl. Leipzig: Reisland.

Becker, Karl Ferdinand (1870): Ausführliche deutsche Grammatik als Kommentar der Schulgrammatik. 2 Bände. 2., neubearb. Ausg. Prag: Tempsky. [Nachdruck Hildesheim: Olms, 1969].

Behaghel, Otto (1923): Deutsche Syntax. Eine geschichtliche Darstellung. Band 1: Die Wortklassen und Wortformen. Heidelberg: Winter.

Betcke, Bruno (1947): Deutscher Sprach-Ratgeber. Leipzig: Brockhaus.

Blatz, Friedrich (1896): Neuhochdeutsche Grammatik mit Berücksichtigung der historischen Entwickelung der deutschen Sprache. Zweiter Band: Satzlehre (Syntax). 3. völlig neubearb. Aufl. Karlsruhe: Lang. [Nachdruck Hildesheim: Olms, 1970].

Brinkmann, Hennig (1971): Die deutsche Sprache. Gestalt und Leistung. 2. neubearb. und erw. Aufl. Düsseldorf: Schwann.

Busse, Dietrich (2006): Sprachnorm, Sprachvariation, Sprachwandel. Überlegungen zu einigen Problemen der sprachwissenschaftlichen Beschreibung des Deutschen im Verhältnis zu seinen Erscheinungsformen. In: Deutsche Sprache 34, S. 314-333.

Duden (2001): Richtiges und gutes Deutsch: Wörterbuch der sprachlichen Zweifelsfälle. 5., neu bearb. u. erw. Aufl. Mannheim: Dudenverlag. (= Duden 9).

Duden (2009): Die Grammatik. Unentbehrlich für richtiges Deutsch. 8., überarb. Aufl. Herausgegeben von der Dudenredaktion. Mannheim u.a.: Dudenverlag (= Duden 4).

Engel, Eduard (1929): Gutes Deutsch: Ein Führer durch Falsch und Richtig. 4. durchges. Aufl. Leipzig: Hesse und Becker.

Engel, Ulrich (1988): Deutsche Grammatik. Heidelberg: Groos.

Erben, Johannes (1965): Abriß der deutschen Grammatik. 8. Aufl. Berlin: Akademie-Verlag.

Erdmann, Oskar/Mensing, Otto (1898): Grundzüge der deutschen Syntax nach ihrer geschichtlichen Entwicklung dargestellt. Stuttgart: Cotta.

Flückiger, Max (1988): Richtiges Deutsch. Berlin: Langenscheidt.

Gottsched, Johann Christoph (1762): Vollständigere und Neuerläuterte Deutsche Sprachkunst. Nach den Mustern der besten Schriftsteller des vorigen und itzigen Jahrhunderts. 5. Aufl., merklich verbessert. Leipzig: Breitkopf. [Nachdruck Hildesheim: Olms, 1970].

Grabarek, Józef (2013). Zur Geschichte der deutschen Sprache im 20. Jahrhundert. Frankfurt/M.: Lang.

Hallwass, Edith (1979): Mehr Erfolg mit gutem Deutsch. Stuttgart u.a.: Das Beste.

Hartweg, Frédéric/Wegera, Klaus-Peter (1989): Frühneuhochdeutsch. Eine Einführung in die deutsche Sprache des Spätmittelalters und der frühen Neuzeit. Tübingen: Niemeyer.

Hirschbold, Karl (1962): Diktieren und Tippen: Gefährliche Klippen im Meer der Grammatik, erfolgreich umsegelt mit Karl Hirschbold. Wien: Jugend und Volk.

Hirschbold, Karl (1976): Besseres Deutsch von A bis Z: Ein Nachschlagewerk für Österreicher. Wien: Österreichischer Bundesverlag für Unterricht, Wissenschaft und Kunst.

Hundt, Markus (2000): „Spracharbeit“ im 17. Jahrhundert. Studien zu Georg Philipp Harsdörffer, Justus Georg Schottelius und Christian Gueintz. Berlin u.a.: de Gruyter.

Jung, Walter (1980): Grammatik der deutschen Sprache. Leipzig: Bibliographisches Institut. 
Keller, R. E. (1986): Die Deutsche Sprache und ihre historische Entwicklung (bearbeitet und übertragen aus dem Englischen von Karl-Heinz Mulagk). Hamburg: Buske.

Konopka, Marek/Strecker, Bruno (Hg.): Deutsche Grammatik - Regeln, Normen, Sprachgebrauch. Berlin u.a.: de Gruyter. (= Jahrbuch des Instituts für Deutsche Sprache 2008).

Löbel, Elisabeth (1986): Apposition und Komposition in der Quantifizierung: Syntaktische, semantische und morphologische Aspekte quantifizierender Nomina im Deutschen. Tübingen: Niemeyer.

Lohnstein, Horst (2014): Artenvielfalt in freier Wildbahn - Generative Grammatik. In: Hagemann, Jörg/ Staffeldt, Sven (Hg.): Syntaxtheorien. Analysen im Vergleich. Tübingen: Stauffenburg. S. 165-185.

Paul, Hermann (1919): Deutsche Grammatik, Band III: Syntax (Erste Hälfte). Tübingen: Niemeyer. [Nachdruck, 1968].

Ratke, Wolfgang (1619): Allgemeine Sprachlehr: Nach der Lehrart Ratichii. Zu Cothen Im Furstenthumb Anhalt.

Schottelius, Justus Georg (1663): Ausführliche Arbeit Von der Teutschen HaubtSprache. (Herausgegeben von Wolfgang Hecht). I. Teil: Bücher I bis III. [Nachdruck Tübingen: Niemeyer, 1967].

Shubina, Elvira (2000): Kvantitativnye slovosochetanija tipa Nquant+AdjN v nemeckom literaturnom jazyke XVII-XIX vekov: osobennosti grammaticheskogo oformlenija i leksicheskogo napolnenija, Dissertation, Moskauer Staatliche Lomonosov-Universität, Moskau.

Shubina, Elvira (2007): Problema normy i variativnosti v nemeckom jazyke na urovne slovosochetanij tipa Nquant+AdjN i eine Art+AdjN (po materialam hudozhestvennoj literatury XVII-XX vekov, sovremennoj pressy i jeksperimental'nyh dannyh), Habilitationsschrift, Moskauer Staatliche Lomonosov-Universität, Moskau.

Trojanskaja, Jelena (1972): Einige Besonderheiten in der Deklination der deutschen Adjektive im 16. und 17. Jahrhundert. In: Studien zur Geschichte der deutschen Sprache. Berlin: Zentralinstitut für Sprachwissenschaft. S. 43-78. (= Bausteine zur Sprachgeschichte des Neuhochdeutschen 19).

Wegera, Klaus-Peter (1985): Morphologie des Frühneuhochdeutschen. In: Besch, Werner/Reichmann, Oskar/Sonderegger, Stefan (Hg.): Sprachgeschichte. Ein Handbuch zur Geschichte der deutschen Sprache und ihrer Erforschung, 2. Halbband., Berlin u.a.: de Gruyter. S. 1313-1322. (= Handbücher zur Sprach- und Kommunikationswissenschaft 2.2).

Wegera, Klaus-Peter (1987): Zur Flexion der Substantive. In: Zeitschrift für deutsche Philologie 106 (Sonderheft: Frühneuhochdeutsch. Zum Stand der sprachwissenschaftlichen Forschung), S. 18-37.

Wells, Christopher J. (1990): Deutsch: eine Sprachgeschichte bis 1945. Tübingen: Niemeyer.

Prof. Dr. Elvira Shubina

Staatliche Moskauer Universität für Internationale Beziehungen (MGIMO)

Prospekt Vernadskogo, 76

RU-119454 Moskau

E-Mail: elvira.shubina@mail.ru

Prof. Dr. Hardarik Blühdorn

Institut für Deutsche Sprache

R 5, 6-13

D-68161 Mannheim

E-Mail: hardarik@ids-mannheim.de 